Preprint typeset in JHEP style - HYPER VERSION

TUM-HEP-671/07

\title{
Challenging SO(10) SUSY GUTs with family symmetries through FCNC processes
}

\author{
Michaela E. Albrecht, Wolfgang Altmannshofer, Andrzej J. Buras, Diego Guadagnoli, \\ and David M. Straub \\ Physik-Department, Technische Universität München, \\ D-85748 Garching, Germany
}

\begin{abstract}
We perform a detailed analysis of the SO(10) SUSY GUT model with $D_{3}$ family symmetry of Dermíšek and Raby (DR). The model is specified in terms of 24 parameters and predicts, as a function of them, the whole MSSM set of parameters at low energy scales. Concerning the SM subset of such parameters, the model is able to give a satisfactory description of the quark and lepton masses, of the PMNS matrix and of the CKM matrix. We perform a global fit to the model, including flavour changing neutral current (FCNC) processes $B_{s} \rightarrow \mu^{+} \mu^{-}, B \rightarrow X_{s} \gamma, B \rightarrow X_{s} \ell^{+} \ell^{-}$and the $B_{d, s}-\bar{B}_{d, s}$ mass differences $\Delta M_{d, s}$ as well as the flavour changing (FC) process $B^{+} \rightarrow \tau^{+} \nu$. These observables provide at present the most sensitive probe of the SUSY mass spectrum and couplings predicted by the model. Our analysis demonstrates that the simultaneous description of the FC observables in question represents a serious challenge for the DR model, unless the masses of the scalars are moved to regions which are problematic from the point of view of naturalness and probably beyond the reach of the LHC. We emphasize that this problem could be a general feature of SUSY GUT models with third generation Yukawa unification and weak-scale minimal flavour violation.
\end{abstract}

KeYwords: GUT, Supersymmetric Standard Model, B-Physics. 


\section{Contents}

1. Introduction 1

2. The model 3

3. Basic procedure 1

3.1 Step 1: Parameters of the model

3.2 Step 2: RG evolution 6

4. One-loop improved determination of low-energy observables 7

5. Basic formulae for FC observables 8

$5.1 B_{s, d} \rightarrow \mu^{+} \mu^{-}$

$5.2 \Delta M_{s, d}$

$5.3 \quad B \rightarrow X_{s} \gamma$

5.4 $B \rightarrow X_{s} \ell^{+} \ell^{-}$

$5.5 \quad B^{+} \rightarrow \tau^{+} \nu$

$5.6(g-2)_{\mu}$

6. General picture 16

7. Numerical analysis

7.1 Fitting procedure 17

7.2 Scenarios 21

7.2.1 $m_{16}=4 \mathrm{TeV}, \mu>0$

$7.2 .2 \quad m_{16}=6 \mathrm{TeV}, \mu>0$

$7.2 .3 \quad m_{16}=10 \mathrm{TeV}, \mu>0$

7.2.4 $m_{16}=4 \mathrm{TeV}, \mu<0$

7.3 Results 23

8. Conclusions 24

\section{Introduction}

A well-known problem of supersymmetry (SUSY) at the electroweak (EW) scale is its proliferation of parameters, arising if one keeps the most general allowed terms in the soft sector. In absence of the latter, SUSY is not phenomenologically viable, while in presence of a most general soft sector, SUSY largely loses its predictivity, due to the bulkiness of the parameter space. 
Since SUSY goes often together with the idea of Grand Unification, due to the tantalizing observation of gauge coupling unification after MSSM running, a sensible way-out to the above problem is to take a 'top-down' approach. In this case one starts with the theory at the GUT scale - with a far simpler parameter space than the MSSM one - and runs all the parameters downwards through Renormalization Group Equations (RGEs). The latter then dynamically generate all the mass splittings for the soft terms at the EW scale, which, in the low-energy MSSM without GUT, are treated instead as free parameters.

As an argument in favour of a top-down approach to the MSSM, it can be noted that predictive SUSY GUT models need typically two ingredients at the GUT scale. The first one is the choice of a specific GUT gauge symmetry. Second, one has to fix the high-scale soft sector with minimal assumptions on the parametric dependence, which seems justified due to a higher amount of symmetry of the theory at this high scale. A further restriction on the number of parameters and hence more predictability of the model can be obtained by introducing additional family symmetries. Once these different parts of the model are specified, the theoretical and computational tools available today allow for a very controlled theoretical error on the model parameters at low-energy, in spite of the 'long running' from the GUT scale and the presence of mass thresholds.

Concerning SUSY GUT models present on the market, while it is easy to construct models reproducing the gauge sector of the MSSM at low-energy, it is far more challenging to find models also correctly describing 'flavour patterns' such as quark and lepton masses and the CKM and PMNS matrices.

One such notable model has been proposed by Dermíšek and Raby in [1]. It is an SO(10) SUSY GUT, augmented with a simple family symmetry, conserving R-parity at low-energy. As shown in [1], this model is able to successfully fit all the parameters of the SM. In particular, by using some observables basically unaffected by SUSY contributions, it can reproduce the CKM matrix entries. Finally, it also describes the known parameters in the neutrino sector. In Ref. [2], the same model was also extensively studied in the lepton flavour sector, thereby providing a number of signatures of the model, which should at least in part be tested by forthcoming experiments.

In view of the above mentioned remarkable performance of the DR model in describing low-energy observables, it is interesting to have a closer look at its SUSY spectrum, with the aim of testing, e.g., the predicted mass hierarchies. Since SUSY particles have not yet been observed, such task can only be accomplished by analyzing implied loop effects in flavour changing neutral current (FCNC) processes. In fact, the specific mass patterns of gauginos, up- and down-squarks and Higgs multiplets predicted by the model, do affect measured quark FCNC observables in a peculiar way, and if such observables are well controlled, the pattern of implied corrections is testable. Fortunately, we have today a whole host of such observables, which have the virtue of being at the same time precisely measured and accurately calculated within the MSSM.

The aim of the present paper is then an in-depth test of the model in the light of all the most accurate information presently available on quark FCNCs. The flavour sector is often overlooked in first analyses of new physics (NP) models, due to its vastness and the necessity of sometimes involved calculations. In the context of the present model, we 
show that the flavour sector has nonetheless enough sensitivity to the details of the SUSY spectrum, to represent a discriminating test for the model itself.

Our paper is organized as follows. In Section 2 we give a brief overview of the DR model, focusing on those ingredients that are most relevant for our purposes. In Section 3 we then outline the procedure to connect the GUT scale model with low-energy observables. Such procedure is well-known, but often obscured by the assumptions made on the running and the mass thresholds. Section 1 goes then in more detail on the determination of masses and couplings, through consideration of low-energy threshold effects. In Section 5 we then present the collection of FCNC observables we use for the purpose of our paper. The emphasis here is on presenting simplified expressions, in order to provide an intuitive picture of the main effects, with refined formulae only used in the actual numerical analysis. In the light of such intuitive expressions, Section 6 then discusses the general pattern featured by the corresponding FCNC observables within the DR model. An extensive analysis of all such features upon variation of the DR model parameters is then presented in Section 7. Finally, Section 8 is devoted to our conclusions.

\section{The model}

The DR model [1, 3] is a supersymmetric $\mathrm{SO}(10)$ Grand Unified Theory with an additional $D_{3} \times\left[U(1) \times Z_{2} \times Z_{3}\right]$ family symmetry.

The above symmetry group fixes the following structure for the superpotential

$$
W=W_{f}+W_{\nu}
$$

with

$$
\begin{aligned}
W_{f} & =\mathbf{1 6}_{3} 1016_{3}+16_{a} 10 \chi_{a} \\
& +\bar{\chi}_{a}\left(M_{\chi} \chi_{a}+45 \frac{\phi_{a}}{\hat{M}} \mathbf{1 6} \mathbf{c}_{3}+\mathbf{4 5} \frac{\tilde{\phi}_{a}}{\hat{M}} \mathbf{1 6}_{a}+A \mathbf{1 6}_{a}\right), \\
W_{\nu} & =\overline{\mathbf{1 6}}\left(\lambda_{2} N_{a} \mathbf{1 6}_{a}+\lambda_{3} N_{3} \mathbf{1 6}_{3}\right)+\frac{1}{2}\left(S_{a} N_{a} N_{a}+S_{3} N_{3} N_{3}\right) .
\end{aligned}
$$

The first two families of quarks and leptons are contained in the superfield $\mathbf{1 6}_{a}, a=$ 1,2 , which transforms under $\mathrm{SO}(10) \times D_{3}$ as $\left(\mathbf{1 6}, 2_{A}\right)$, whereas the third family in $\mathbf{1 6}_{3}$ transforms as $\left(\mathbf{1 6}, 1_{B}\right)$. The two MSSM Higgs doublets $H_{u}$ and $H_{d}$ are contained in a 10. As can be seen from the first term on the right-hand side of (2.1), Yukawa unification $\lambda_{t}=\lambda_{b}=\lambda_{\tau}=\lambda_{\nu_{\tau}}$ at $M_{G}$ is obtained only for the third generation, which is directly coupled to the Higgs 10 representation. This immediately implies large $\tan \beta \approx 50$ at low energies.

The effective Yukawa couplings of the first and second generation fermions are instead generated via the Froggatt-Nielsen mechanism [4] as follows. Additional fields are introduced, i.e. the $\mathbf{4 5}$ which is an adjoint of $\mathrm{SO}(10)$, the $\mathrm{SO}(10)$ singlet flavon fields $\phi^{a}, \tilde{\phi}^{a}, A$ and the Froggatt-Nielsen states $\chi_{a}, \bar{\chi}_{a}$. The latter transform as a $\left(\mathbf{1 6}, 2_{A}\right)$ and a $\left(\overline{\mathbf{1 6}}, 2_{A}\right)$, respectively, and receive masses of $\mathrm{O}\left(M_{G}\right)$ as $M_{\chi}$ acquires an $\mathrm{SO}(10)$ breaking VEV. Once 
they are integrated out, they give rise to effective mass operators which, together with the VEVs of the flavon fields, create the Yukawa couplings for the first two generations. This mechanism breaks systematically the full flavour symmetry and produces the right mass hierarchies among the fermions.

The obtained Yukawa matrices for up-quarks, down-quarks, charged leptons and neutrinos are

$$
\begin{gathered}
Y_{u}=\left(\begin{array}{ccc}
0 & \varepsilon^{\prime} \rho-\varepsilon \xi \\
-\varepsilon^{\prime} \rho & \tilde{\varepsilon} \rho & -\varepsilon \\
\varepsilon \xi & \varepsilon & 1
\end{array}\right) \lambda, \quad Y_{d}=\left(\begin{array}{ccc}
0 & \varepsilon^{\prime} & -\varepsilon \xi \sigma \\
-\varepsilon^{\prime} & \tilde{\varepsilon} & -\varepsilon \sigma \\
\varepsilon \xi & \varepsilon & 1
\end{array}\right) \lambda, \\
Y_{e}=\left(\begin{array}{ccc}
0 & -\varepsilon^{\prime} & 3 \varepsilon \xi \\
\varepsilon^{\prime} & 3 \tilde{\varepsilon} & 3 \varepsilon \\
-3 \varepsilon \xi \sigma & -3 \varepsilon \sigma & 1
\end{array}\right) \lambda, \quad Y_{\nu}=\left(\begin{array}{ccc}
0 & -\varepsilon^{\prime} \omega & \frac{3}{2} \varepsilon \xi \omega \\
\varepsilon^{\prime} \omega & 3 \tilde{\varepsilon} \omega & \frac{3}{2} \varepsilon \omega \\
-3 \varepsilon \xi \sigma-3 \varepsilon \sigma & 1
\end{array}\right) \lambda .
\end{gathered}
$$

From eqs. (2.3) one can see that the flavour hierarchies in the Yukawa couplings are encoded in terms of the four complex parameters $\rho, \sigma, \tilde{\varepsilon}, \xi$ and the additional real ones $\varepsilon, \varepsilon^{\prime}, \lambda$.

In order to avoid neutrino masses of the order of the other fermion masses, one invokes the type-I see-saw mechanism [5, 6, 6, 8]. In particular, three $\mathrm{SO}(10)$ singlet Majorana fermion fields $N_{a}, N_{3}(a=1,2)$ are introduced via the contribution of $\frac{1}{2}\left(S_{a} N_{a} N_{a}+S_{3} N_{3} N_{3}\right)$ to the superpotential.

The mass term $\frac{1}{2} N M_{N} N$ is produced when the flavon fields acquire VEVs $\left\langle S_{a}\right\rangle=M_{N_{a}}$ and $\left\langle S_{3}\right\rangle=M_{N_{3}}$. Together with a $\overline{\mathbf{1 6}}$ Higgs one is allowed to introduce the interaction terms $\overline{\mathbf{1 6}}\left(\lambda_{2} N_{a} \mathbf{1 6}_{a}+\lambda_{3} N_{3} \mathbf{1 6}_{3}\right)$, which in turn generate a mixing matrix $V$ between the right-handed neutrinos and the additional singlets $(\bar{\nu} V N)$, when the $\overline{\mathbf{1 6}}$ acquires an $\mathrm{SO}(10)$ breaking $\operatorname{VEV}\langle\overline{\mathbf{1 6}}\rangle_{\bar{\nu}}=v_{16}$. The resulting effective right-handed neutrino mass terms read

$$
\begin{gathered}
W_{N}=\bar{\nu} V N+\frac{1}{2} N M_{N} N \\
V=v_{16}\left(\begin{array}{ccc}
0 & \lambda_{2} & 0 \\
\lambda_{2} & 0 & 0 \\
0 & 0 & \lambda_{3}
\end{array}\right), \quad M_{N}=\operatorname{diag}\left(M_{N_{1}}, M_{N_{2}}, M_{N_{3}}\right) .
\end{gathered}
$$

Diagonalization leads to the effective right-handed neutrino Majorana mass

$$
M_{R}=-V M_{N}^{-1} V^{T} \equiv-\operatorname{diag}\left(M_{R_{1}}, M_{R_{2}}, M_{R_{3}}\right) .
$$

By integrating out the EW singlets $\bar{\nu}$ and $N$, which both receive GUT scale masses, one ends up with the light neutrino mass matrix at the EW scale given by the usual see-saw formula

$$
\mathcal{M}=m_{\nu} M_{R}^{-1} m_{\nu}^{T}
$$

\section{Basic procedure}

In this section, we describe the procedure which, from the specification of the model at the GUT scale, leads to the MSSM mass spectrum and observables at the EW scale. 


\subsection{Step 1: Parameters of the model}

\section{Gauge coupling sector}

We choose as three parameters the unification scale $M_{G}$, the gauge coupling $\alpha_{G}$ defined through

$$
\alpha_{G} \equiv \alpha_{1}\left(M_{G}\right)=\alpha_{2}\left(M_{G}\right)
$$

and the threshold correction $\epsilon_{3}$ defined through

$$
\alpha_{3}\left(M_{G}\right)=\alpha_{G}\left(1+\epsilon_{3}\right) .
$$

The threshold correction $\epsilon_{3}$ helps to obtain the right value of $\alpha_{3}\left(M_{Z}\right)$.

\section{SUSY sector}

We have the following set of soft SUSY breaking parameters: a universal sfermion mass $m_{16}$, a universal gaugino mass $M_{1 / 2}$, a universal trilinear coupling parameter $A_{0}$, and the Higgs mass parameters $m_{H_{u}}$ and $m_{H_{d}}$. It is well-known that at large $\tan \beta$, EWSB is easier to achieve by allowing the soft SUSY breaking Higgs mass parameters to be split. This also has the consequence that the absolute value of $\mu$ is not fixed by EWSB, as is the case in the $\mathrm{CMSSM}^{1}$, but is instead a free parameter.

\section{Yukawa matrices}

The Yukawa matrices for up- and down-type quarks, charged leptons and neutrinos are parameterized as given in (2.3), with $\varepsilon, \varepsilon^{\prime}, \lambda$ being real and $\rho, \sigma, \tilde{\varepsilon}, \xi$ complex parameters.

\section{Right-handed neutrinos}

The diagonal right-handed neutrino mass matrix is chosen to be real, which amounts to three parameters $M_{R_{i}}$, with $i=1,2,3$.

\section{Weak-scale parameters}

In addition to the above GUT-scale parameters, the SUSY parameters $\mu$ and $\tan \beta$ have also to be specified as an input at the weak scale.

The total number of input parameters, listed in this step and summarized in Table 1, is therefore 24. Once these parameters are fixed, the MSSM couplings as well as its whole mass spectrum (which includes the SM part) can be predicted at energies below $M_{G}$, in particular at $M_{Z}$ or any lower scale relevant for FCNC processes. Of course a subset of the observables amenable to prediction is used to fix the model parameters.

The procedure to fix the above parameters will be addressed in detail in Section 7 . Such procedure uses low-energy observables, and for this reason we need to evolve the fundamental parameters of the theory to $M_{Z}$ or below, using their RGEs. The evolution to low energy is performed according to a procedure we will now describe.

\footnotetext{
${ }^{1}$ Very interesting combined analyses in the framework of the CMSSM have been performed in 9 , 10, 11. In [12], also non-universal Higgs masses were considered, but only with vanishing $A_{0}$.
} 


\begin{tabular}{|lcc|}
\hline Sector & $\#$ & Parameters \\
\hline \hline gauge & 3 & $\alpha_{G}, M_{G}, \epsilon_{3}$, \\
SUSY (GUT scale) & 5 & $m_{16}, M_{1 / 2}, A_{0}, m_{H_{u}}, m_{H_{d}}$, \\
textures & 11 & $\epsilon, \epsilon^{\prime}, \lambda, \rho, \sigma, \epsilon, \xi$, \\
neutrino & 3 & $M_{R_{1}}, M_{R_{2}}, M_{R_{3}}$, \\
SUSY (EW scale) & 2 & $\tan \beta, \mu$ \\
\hline \hline
\end{tabular}

Table 1: Parameters in the DR model.

\subsection{Step 2: RG evolution}

\section{$M_{\mathrm{GUT}}>$ scale $>M_{R_{i}}$}

The RGEs of the MSSM with right-handed neutrinos [13, 14, 15] are used to run the gauge couplings, Yukawa couplings, all soft SUSY breaking parameters and the right-handed neutrino mass matrix $M_{R}$ down to the mass of the heaviest right-handed neutrino. At this threshold, one must rediagonalize $M_{R}$ and integrate out one neutrino following [16]. This generates the effective dimension-five neutrino mass operator. The two remaining thresholds are treated accordingly, with the number of neutrinos reduced by one each time. In practical calculations, this procedure turns out to be computationally demanding. We have thus followed the approach of integrating out all the right-handed neutrinos at a single intermediate threshold, corresponding to the mass of the lightest of them. We have then checked that this approximate treatment does not have any relevant impact on either the determination of the GUT-scale parameters or the low-energy predictions of the model.

\section{$M_{R_{i}}>$ scale $>M_{Z}$}

Having constructed the effective theory without right-handed neutrinos at the scale where they are integrated out, we use MSSM RGEs [17] to run the gauge couplings, Yukawa matrices, soft SUSY breaking parameters and the Wilson coefficient of the neutrino mass operator down to the scale $M_{Z}$. We use two-loop RGEs for dimensionless and one-loop for dimensionful parameters.

So, starting from the fundamental parameters of Step 1, and performing RGE evolution through Step 2, one has now the whole set of parameters of the MSSM fixed at $M_{Z}$ or below, to tree-level. However, for many quantities, such as gauge couplings, some SUSY masses, quark masses, CKM matrix and the Higgs sector, a one-loop determination turns out to be mandatory, for different reasons. These issues will be discussed in the next section.

Here we would like to stress a further property of the low-scale MSSM parameters implied by the DR model. The off-diagonal entries in the squark mass matrices are generated radiatively by the Yukawa couplings; in addition the quark sector features only one CP phase ${ }^{2}$. These two facts allow to classify the low-energy MSSM resulting from the

\footnotetext{
${ }^{2}$ Putting additional phases in the right-handed neutrino mass matrix would communicate corresponding phases to the low-energy neutrino sector, without affecting, to a very good approximation, the quark sector.
} 
RG evolution of the DR model as belonging to the class of models with minimal flavour violation (MFV) [18].

With the above qualifications, using the numerically determined parameters of the theory, we can then evaluate effective Hamiltonians for weak decays, in particular for FCNC and CP-violating processes. Having these Hamiltonians at hand, it is straightforward to evaluate the branching ratios for various low energy processes and to compare them with experiments. The latter strategy, allowing to extensively test the model in the flavour sector, will be dealt with in Sections 6 and 7 .

\section{One-loop improved determination of low-energy observables}

With low-energy observables we denote all the physical quantities that can be directly accessed experimentally and compared with predictions of the model. Among these quantities, some will be used to fix the model parameters, through a fitting procedure, as described in Section 7. The others will then be genuine predictions of the model.

To obtain all physical observables beyond tree-level accuracy at the scale $M_{Z}$, one needs to include one-loop corrections in the relevant formulae defining the observables themselves. The inclusion of such corrections is also advocated in the analyses [1], 2]. Below, we give some details of the procedure in the various cases.

\section{SUSY masses}

The mass eigenstates for squarks, sleptons, charginos and neutralinos are calculated at the weak scale from the tree-level mass matrices [19]. The gluino pole mass is calculated at the one-loop level.

\section{Gauge sector}

We calculate the gauge couplings $\alpha_{s}\left(M_{Z}\right)$ and $\alpha_{\mathrm{em}}$ by including the threshold corrections given in [20]. We use tadpole corrections to the Higgs potential [20, 21] to obtain the one-loop VEV and include one-loop SUSY corrections to the Fermi constant measured in muon decay, $G_{\mu}$, as well as to the $W$ and $Z$ boson pole masses [21].

\section{Higgs spectrum}

The Higgs spectrum is represented by the masses $M_{h_{0}}, M_{H_{0}}, M_{H^{+}}, M_{A}$ of the corresponding physical particles. In this model $H_{0}, H^{+}$and $A$ are typically nearly degenerate, with only the lightest Higgs mass $M_{h_{0}}$ lying at a substantially lower value. The scale of the masses $M_{H_{0}}, M_{H^{+}}, M_{A}$ is set in a non-trivial way by the interplay among $\mu, \tan \beta, m_{H_{u, d}}$ and $m_{16}$ in the equations minimizing the Higgs potential.

We calculate the pseudoscalar Higgs pole mass $M_{A}$ following [20] and use it as an input to FeynHiggs 2.5.1 [22, 23, 24, 25], which accurately calculates the masses of the remaining Higgs mass eigenstates, using Yukawa matrices and soft terms at the EW scale, which in turn are the result of the RG analysis.

We explicitly note that $M_{A}$ (and with it the other heavy Higgs masses) is typically 'pushed up' by the upper bound on the $\operatorname{BR}\left(B_{s} \rightarrow \mu^{+} \mu^{-}\right)$, so that the $M_{A}$ value obtained 
with any given choice of the rest of the parameters can be considered as a lower mass bound on the heavy Higgs spectrum.

\section{Fermion masses and CKM matrix}

At large $\tan \beta$, quark masses undergo $\tan \beta$ enhanced corrections that have to be included to the tree-level determination, represented by the running Yukawa couplings at $M_{Z}$ [26]. These corrections also modify the relations between the original CKM matrix appearing in the MSSM Feynman rules and the effective CKM matrix measured in tree-level decays [27, 28]. We closely follow the line of argument of [28] and calculate the one-loop threshold corrections to quark and charged lepton mass matrices at $M_{Z}$, but take into account both SUSY and electroweak contributions. After applying the threshold corrections, we use three-loop QCD and one-loop QED RGEs to run the five light quark and three charged lepton masses down to their respective scales.

The neutrino masses and the PMNS mixing matrix, on the other hand, are left at the tree-level. In fact we find the threshold corrections to these quantities, discussed in [29], to be numerically negligible.

\section{Basic formulae for $\mathrm{FC}$ observables}

In this section we collect formulae for various branching ratios that we will use in our numerical analysis. In certain cases we show only the leading contribution for large $\tan \beta$, in order to provide an intuitive picture of the behaviour. For example, in the case of $B_{s, d} \rightarrow \mu^{+} \mu^{-}$decays, this corresponds to the contribution of Higgs penguins. In the actual numerical analysis we include all the relevant contributions, i.e., besides the SM one, contributions from charginos, charged Higgses and gluinos. Neutralino contributions are generally negligible.

\section{$5.1 B_{s, d} \rightarrow \mu^{+} \mu^{-}$}

In the SM, the usual $Z$-penguin and box diagrams result in strongly suppressed branching ratios that are sensitive functions of the weak decay constants $F_{B_{d}}$ and $F_{B_{s}}$. Eliminating this dependence with the help of the well-measured mass differences $\Delta M_{s, d}$ [30], one finds 31.

$$
\begin{aligned}
& \mathrm{BR}\left(B_{s} \rightarrow \mu^{+} \mu^{-}\right)_{\mathrm{SM}}=(3.37 \pm 0.31) \times 10^{-9}, \\
& \mathrm{BR}\left(B_{d} \rightarrow \mu^{+} \mu^{-}\right)_{\mathrm{SM}}=(1.02 \pm 0.09) \times 10^{-10}
\end{aligned}
$$

These values should be compared with the present 95\% C.L. upper bounds from CDF 32

$$
\mathrm{BR}\left(B_{s} \rightarrow \mu^{+} \mu^{-}\right)_{\exp }<1.0 \times 10^{-7}, \quad \mathrm{BR}\left(B_{d} \rightarrow \mu^{+} \mu^{-}\right)_{\exp }<3.0 \times 10^{-8},
$$

that leave still a large room for NP contributions.

In the MSSM with large $\tan \beta$, the helicity suppression in (5.1) and (5.2) is lifted by Higgs-mediated neutral currents [33, 34]. Their contributions can be summarized by the 
approximate formula 28, 35]

$$
\begin{aligned}
\operatorname{BR}\left(B_{s} \rightarrow \mu^{+} \mu^{-}\right) & \simeq 3.5 \times 10^{-5}\left[\frac{\tan \beta}{50}\right]^{6}\left[\frac{\tau_{B_{s}}}{1.5 \mathrm{ps}}\right]\left[\frac{F_{B_{s}}}{230 \mathrm{MeV}}\right]^{2}\left[\frac{\left|V_{t s}\right|}{0.040}\right]^{2} \\
& \times \frac{\bar{m}_{t}^{4}}{M_{A}^{4}} \frac{\left(16 \pi^{2} \epsilon_{Y}\right)^{2}}{\left(1+\tilde{\epsilon}_{3} \tan \beta\right)^{2}\left(1+\epsilon_{0} \tan \beta\right)^{2}}
\end{aligned}
$$

where $\bar{m}_{t} \equiv m_{t}\left(\mu_{t}\right)$ and

$$
\tilde{\epsilon}_{3}=\epsilon_{0}+y_{t}^{2} \epsilon_{Y}
$$

with $\epsilon_{0}$ and $\epsilon_{Y}$ standing for gluino loop and chargino loop factors, whose full expressions can be found in [28].

With a similar expression for $\mathrm{BR}\left(B_{d} \rightarrow \mu^{+} \mu^{-}\right)$, one also gets

$$
\frac{\mathrm{BR}\left(B_{d} \rightarrow \mu^{+} \mu^{-}\right)}{\operatorname{BR}\left(B_{s} \rightarrow \mu^{+} \mu^{-}\right)}=\left[\frac{\tau_{B_{d}}}{\tau_{B_{s}}}\right]\left[\frac{F_{B_{d}}}{F_{B_{s}}}\right]^{2}\left[\frac{\left|V_{t d}\right|}{\left|V_{t s}\right|}\right]^{2}\left[\frac{M_{B_{d}}}{M_{B_{s}}}\right]^{5}
$$

where non-leading contributions have been neglected.

Observing that the ratio (5.6) is roughly a factor of ten smaller than the corresponding ratio of experimental bounds (5.3), it is clear that, in our framework, the current $\operatorname{BR}\left(B_{d} \rightarrow\right.$ $\mu^{+} \mu^{-}$) constraint is completely marginal with respect to the $B_{s}$ counterpart, which is the only channel considered in the rest of the analysis.

We explicitly note that, in eq. (5.4), as throughout the text, $V_{t i}$ denotes elements of the physical CKM matrix ${ }^{3}$, to be distinguished for large $\tan \beta$ from the corresponding matrix appearing at the Lagrangian level. The latter is denoted as 'bare' since it does not yet include the large $\tan \beta$-resummed effects. A similar comment applies to the quark masses. In actual numerical calculations, the differences between the physical and the 'bare' parameters have been taken into proper account as discussed in section 4 .

At this stage, it suffices to state that $\epsilon_{0}$ and $\tilde{\epsilon}_{3}$ are at most $\mathrm{O}\left(10^{-2}\right)$. Still, with $\tan \beta \approx 50$, as characteristic for the DR model, the $\tan \beta$-resummed corrections in the last factor in (5.4) can be significant and, depending on the sign of $\mu$, can provide an additional enhancement of $\operatorname{BR}\left(B_{s} \rightarrow \mu^{+} \mu^{-}\right)$or respectively some suppression relative to the leading behaviour $(\tan \beta)^{6}$. One finds in the full space of parameters considered

$$
\operatorname{sign}\left(\epsilon_{0}\right)=\operatorname{sign}\left(\tilde{\epsilon}_{3}\right)=\operatorname{sign}(\mu)
$$

and more explicitly,

$$
\begin{aligned}
\epsilon_{0} & \approx-\frac{2 \alpha_{s}}{3 \pi} \frac{\mu}{m_{\tilde{g}}} H_{2}\left(m_{\tilde{b}_{1}}^{2} / m_{\tilde{g}}^{2}, m_{\tilde{b}_{2}}^{2} / m_{\tilde{g}}^{2}\right), \\
\epsilon_{Y} & \approx \frac{1}{16 \pi^{2}} \frac{A_{t}}{\mu} H_{2}\left(m_{\tilde{t}_{1}}^{2} / \mu^{2}, m_{\tilde{t}_{2}}^{2} / \mu^{2}\right),
\end{aligned}
$$

\footnotetext{
${ }^{3}$ In the notation of 28$]$, such matrix elements are written as $V_{t i}^{\text {eff }}$.
} 
where $m_{\tilde{b}_{i}}\left(m_{\tilde{t}_{i}}\right)$ are the masses of the $i^{\text {th }}$ sbottom (stop), $A_{t}$ is the soft SUSY breaking stop trilinear parameter ${ }^{4}$ and $m_{\tilde{g}}$ the gluino mass. The function $\mathrm{H}_{2}$ is defined as [28] (see also [36])

$$
H_{2}(x, y)=\frac{x \log x}{(1-x)(x-y)}+\frac{y \log y}{(1-y)(y-x)} .
$$

We emphasize that in the DR model the parameters entering $\epsilon_{0}$ and $\epsilon_{Y}$ are strongly correlated with each other and consequently the range of values which $\epsilon_{0}$ and $\epsilon_{Y}$ can take is significantly smaller than in the usual studies of the above formulae, that can be found in the literature.

We also emphasize that in the numerical analysis one can replace the above branching ratio with the quantity [30]

$$
\frac{\operatorname{BR}\left(B_{s} \rightarrow \mu^{+} \mu^{-}\right)}{\Delta M_{s}}
$$

thereby eliminating $F_{B_{s}}$ that is still inaccurately known. The only hadronic uncertainties in the ratio in (5.11) are present in the non-perturbative factors $B_{s}^{i}$, that enter $\Delta M_{s}$ only linearly and are better known from lattice calculations than the decay constant 37.

\section{$5.2 \Delta M_{s, d}$}

The mass differences in the $B_{s, d}-\bar{B}_{s, d}$ systems, $\Delta M_{s, d}$, consist, in the MSSM at large $\tan \beta$, of the following contributions

$$
\Delta M_{s, d}=\Delta M_{s, d}^{\mathrm{SM}}+\Delta M_{s, d}^{H^{+}}+\Delta M_{s, d}^{\tilde{\chi}^{+}}+\Delta M_{s, d}^{\tilde{g}}+\Delta M_{s, d}^{\tilde{g} \tilde{\chi}^{0}}+\Delta M_{s, d}^{\tilde{\chi}^{0}}+\Delta M_{s, d}^{\mathrm{DP}},
$$

i.e. box diagrams with the SM contribution, with charged Higgses, charginos, gluinos, gluino-neutralino and neutralinos and finally neutral Higgs double-penguins, respectively. Explicit formulae for $\Delta M_{s, d}$ that include all the important contributions are given in $[28] .^{5}$ The values of $B_{s, d}^{i}$ are taken from [37]. Besides the dominant SM contribution, the most important NP contributions in the DR model are $\Delta M_{s, d}^{\tilde{\chi}^{+}}$and especially $\Delta M_{s, d}^{\mathrm{DP}}$. The latter is strictly negative [28]

$$
\begin{gathered}
\Delta M_{s}^{\mathrm{DP}}=-12.0 \mathrm{ps}^{-1}\left[\frac{\tan \beta}{50}\right]^{4}\left[\frac{F_{B_{s}}}{230 \mathrm{MeV}}\right]^{2}\left[\frac{\left|V_{t s}\right|}{0.040}\right]^{2} \\
\times\left[\frac{\bar{m}_{b}\left(\mu_{t}\right)}{3.0 \mathrm{GeV}}\right]\left[\frac{\bar{m}_{s}\left(\mu_{t}\right)}{0.06 \mathrm{GeV}}\right]\left[\frac{\bar{m}_{t}^{4}\left(\mu_{t}\right)}{M_{W}^{2} M_{A}^{2}}\right] \frac{\left(16 \pi^{2} \epsilon_{Y}\right)^{2}}{\left(1+\tilde{\epsilon}_{3} \tan \beta\right)^{2}\left(1+\epsilon_{0} \tan \beta\right)^{2}} .
\end{gathered}
$$

\footnotetext{
${ }^{4}$ Our sign convention for $A_{t}$ is such that the off-diagonal entry of the tree-level stop mass matrix reads $m_{t}\left(A_{t}-\mu \cot \beta\right)$. This agrees with the sign convention for $A_{0}$ in [1], 2] but disagrees with the convention used in [19, 28].

${ }^{5}$ We mention that gluino and neutralino box contributions were not considered in 28. In fact, we find these contributions to be very small, but still included them in our numerical analysis. We did not include, instead, subleading effects in the Higgs propagator appearing in DP diagrams. The latter have been recently addressed in [38] and play an insignificant role in the present analysis.
} 
We recall that, experimentally [39],

$$
\left(\Delta M_{s}\right)_{\exp }=(17.77 \pm 0.10 \pm 0.07) / \mathrm{ps}
$$

to be compared with the UTfit and CKMfitter SM predictions 40, 41]

$$
\left.\left(\Delta M_{s}\right)\right)_{\mathrm{UTfit}}^{\mathrm{SM}}=(18.6 \pm 2.3) / \mathrm{ps}, \quad\left(\Delta M_{s}\right)_{\mathrm{CKMfitter}}^{\mathrm{SM}}=\left(18.9_{-2.8}^{+5.9}\right) / \mathrm{ps} .
$$

The CKMfitter result still allows for very sizeable NP contributions, while the UTfit result bounds $\left|\Delta M_{s}\right|^{\text {DP }}$ to be below $\approx 3 /$ ps provided other NP contributions in (5.12) can be neglected. We will see in Section 7 that in the DR model $\Delta M_{s}$ is slightly suppressed relative to the SM, but this suppression amounts to at most $5 \%$, in accordance with experimental findings.

Concerning $\Delta M_{d}^{\mathrm{DP}}$, it is suppressed by at least two orders of magnitude relative to $\Delta M_{s}^{\mathrm{DP}}$ due to $m_{d} / m_{s}$ and $\left|V_{t d}\right|^{2} /\left|V_{t s}\right|^{2}$ factors and consequently in the DR model $\Delta M_{d}$ is SM-like to a very good accuracy.

Finally, we note the role played in the present model by the strong correlation 42 between the Higgs penguin contributions to $\operatorname{BR}\left(B_{s, d} \rightarrow \mu^{+} \mu^{-}\right)$and $\Delta M_{s}$ : the enhancement of $\operatorname{BR}\left(B_{s, d} \rightarrow \mu^{+} \mu^{-}\right)$in the DR model is correlated with a suppression of $\Delta M_{s}$. However, the data on $\Delta M_{s}$ do not allow for very large enhancements of $\operatorname{BR}\left(B_{s, d} \rightarrow \mu^{+} \mu^{-}\right)$so that both observables turn into constraints on the pseudoscalar Higgs mass $M_{A}$. We will come back to this point in Section 6 .

\section{$5.3 B \rightarrow X_{s} \gamma$}

An important constraint on any NP model is the inclusive decay $B \rightarrow X_{s} \gamma$ for which the data read 43, 44, 45]

$$
\mathrm{BR}\left(B \rightarrow X_{s} \gamma\right)_{\exp }=(3.55 \pm 0.24 \pm 0.10 \pm 0.03) \times 10^{-4}
$$

to be compared with the SM value at the NNLO level [46]

$$
\mathrm{BR}\left(B \rightarrow X_{s} \gamma\right)_{\mathrm{SM}}=(3.15 \pm 0.23) \times 10^{-4} .
$$

The inclusion of certain non-perturbative effects decreases this value to $(2.98 \pm 0.26) \times$ $10^{-4}$ [47. The latter value, if confirmed, would put some tension on the SM prediction. In any case, unless the central experimental value in (5.15) will be significantly decreased, NP scenarios predicting $\operatorname{BR}\left(B \rightarrow X_{s} \gamma\right)$ to be smaller than the SM value are disfavoured.

Instead of presenting detailed formulae for $B \rightarrow X_{s} \gamma$ in the DR model, which can be found in the literature [48, 49, 50, 18], we collect here a number of qualitative properties of these formulae that will turn out to be useful in understanding our numerical results. These properties are as follows:

- The charged and neutral Higgs contributions to $\operatorname{BR}\left(B \rightarrow X_{s} \gamma\right)$ are strictly positive.

- The sign of the chargino contributions relative to the SM is ruled by the following relation

$$
C_{7}^{\tilde{\chi}^{+}} \propto+\mu A_{t} \tan \beta \times \operatorname{sign}\left(C_{7}^{\mathrm{SM}}\right)
$$


with a positive proportionality factor, so it is opposite to that of the SM one for $\mu>0$ and $A_{t}<0$ (cf. footnote 1 ).

This shows that the large $\tan \beta$ effects in $B \rightarrow X_{s} \gamma$ are not as strong as in $B_{s} \rightarrow \mu^{+} \mu^{-}$, where the amplitude behaves as $\tan ^{3} \beta / M_{A}^{2}$. However, they are typically more important than in $\Delta M_{s}$, since in the latter case contributions of Higgs penguins, while behaving as $\tan ^{4} \beta / M_{A}^{2}$, are suppressed by the ratio of the external quark masses over $M_{W}^{2}$.

Among the NP contributions to $B \rightarrow X_{s} \gamma$, those from charginos are generically the largest. In fact, the lightest chargino mass is roughly set by the lowest between $\mu$ and $M_{2}$ and in the DR model it turns out to be generically below $\approx 200 \mathrm{GeV}$. On the other hand, Higgs contributions are generically small in the DR model, since $M_{A, H^{+}}$are pushed up by the $B_{s} \rightarrow \mu^{+} \mu^{-}$constraint. Consequently, for positive $\mu$, the sign of the Wilson coefficient $C_{7}$ can be reversed relative to $C_{7}^{\mathrm{SM}}$, while $\Delta M_{s}$, as stated above, cannot be modified by more than $\approx 5 \%$ if the constraint from $B_{s} \rightarrow \mu^{+} \mu^{-}$is taken into account.

Indeed, for positive $\mu$, in order to pass the $B \rightarrow X_{s} \gamma$ constraint, the model favours the solution $C_{7}\left(\mu_{b}\right)=-C_{7}^{\mathrm{SM}}\left(\mu_{b}\right)$. We stress here that such solution is a highly conspired one, since such equality should hold at the $\mu_{b}$ scale, i.e. after running of the coefficients from the matching scale. In addition, in this case SUSY is not quite a correction to the SM result, but rather the opposite. As a consequence, to address this case, one would need a theoretical control on the SUSY part at least as good as that on the pure SM calculation. This task is in turn very hard to achieve, since e.g., one would have to accurately know where to integrate out the various sectors of the MSSM entering the SUSY contributions to $B \rightarrow X_{s} \gamma$. In absence of such knowledge, one can take the approach of matching the whole SUSY spectrum at a common reasonable scale. This approach works if SUSY is a correction to the SM. But in the present case, the solution $C_{7}\left(\mu_{b}\right)=-C_{7}^{\mathrm{SM}}\left(\mu_{b}\right)$ is extremely sensitive to variation on the matching scale, and the theoretical error associated completely out of control. We will come back to this point in section 6 .

\section{$5.4 B \rightarrow X_{s} \ell^{+} \ell^{-}$}

An important observable in our analysis will be the branching ratio for the inclusive decay $B \rightarrow X_{s} \ell^{+} \ell^{-}$and the related forward-backward asymmetry $A_{\mathrm{FB}}$. A significant progress in calculating this decay and its exclusive counterpart $B \rightarrow K^{*} \ell^{+} \ell^{-}$has been achieved in recent years through the calculation of the NNLO QCD corrections. The corresponding formulae are very complicated and will not be presented here. They can be found in [51, 52, 53, 54, 55, 56, 57, 58, 59]. For our discussion it will be sufficient to recall the NLO formulae 60, 61], keeping only the dipole operator and the operators

$$
Q_{9}=(\bar{s} b)_{V-A}(\bar{\mu} \mu)_{V}, \quad Q_{10}=(\bar{s} b)_{V-A}(\bar{\mu} \mu)_{A}
$$

The contributions of semi-leptonic scalar operators to this decay are much less important than in $B_{d, s} \rightarrow \mu^{+} \mu^{-}$, since $B \rightarrow X_{s} \ell^{+} \ell^{-}$is not helicity suppressed. We have taken them into account following [62], but their inclusion in the present discussion would only complicate matters without changing the basic picture. 
Introducing the normalized dilepton mass parameter

$$
\hat{s}=\frac{\left(p_{\mu^{+}}+p_{\mu^{-}}\right)^{2}}{m_{b}^{2}} \equiv \frac{s}{m_{b}^{2}},
$$

the invariant dilepton mass spectrum in the inclusive decay $B \rightarrow X_{s} \ell^{+} \ell^{-}$is roughly given at NLO as follows

$$
\frac{d \Gamma\left(B \rightarrow X_{s} \ell^{+} \ell^{-}\right)}{d \hat{s}} \sim(1-\hat{s})^{2}\left|V_{t s}\right|^{2} U(\hat{s})
$$

where

$$
U(\hat{s})=(1+2 \hat{s})\left(\left|\tilde{C}_{9}^{\mathrm{eff}}(\hat{s})\right|^{2}+\left|\tilde{C}_{10}\right|^{2}\right)+4\left(1+\frac{2}{\hat{s}}\right)\left|C_{7}^{(0) \mathrm{eff}}\right|^{2}+12 C_{7}^{(0) \mathrm{eff}} \operatorname{Re}\left(\tilde{C}_{9}^{\mathrm{eff}}(\hat{s})\right)
$$

with the MSSM expression for the Wilson coefficients $C_{7}$ [48] and the SM ones for $C_{9}, C_{10}$ given in [60, 61, 63] ${ }^{6}$.

Of particular interest is the (normalized) forward-backward asymmetry in $B \rightarrow X_{s} \ell^{+} \ell^{-}$. It becomes non-zero only at the NLO level. It is given in this approximation as follows 54

$$
\bar{A}_{\mathrm{FB}}(\hat{s})=-3 \operatorname{Re}\left[\tilde{C}_{10}^{*} \frac{\hat{s} \tilde{C}_{9}^{\mathrm{eff}}(\hat{s})+2 C_{7}^{(0) \mathrm{eff}}}{U(\hat{s})}\right] .
$$

The expression for the corresponding asymmetry in the exclusive decay $B \rightarrow K^{*} \ell^{+} \ell^{-}$ can be found in [58, 59]. Both asymmetries vanish in the SM at a certain $\hat{s}=\hat{s}_{0}$ [66], which in the case of the inclusive decay is determined through

$$
\hat{s}_{0} \operatorname{Re} \tilde{C}_{9}^{\mathrm{eff}}\left(\hat{s}_{0}\right)+2 C_{7}^{(0) \mathrm{eff}}=0 .
$$

In the $\mathrm{SM}$ at $\mathrm{NLO}$ one finds $\hat{s}_{0} \approx 0.14$. At NNLO the corresponding value is $\hat{s}_{0}=$ $0.162 \pm 0.008$ [52, 53, 67, 68, 54].

Now, as can be seen in (5.21), the very low- $s$ region $\left(s<1 \mathrm{GeV}^{2}\right)$ is dominated by the coefficient $C_{7}$ and does not provide more information than already contained in the $B \rightarrow X_{s} \gamma$ decay. Much more useful is then the low-s region $\left(1 \mathrm{GeV}^{2}<s<6 \mathrm{GeV}^{2}\right)$ which is theoretically cleaner than the high-s region, is dominated by the Wilson coefficients $C_{9}$ and $C_{10}$ and is also very sensitive to the $C_{7}-C_{9}$ interference in (5.21). For this low- $s$ range the world average coming from Belle [69] and BaBar [70] reads

$$
\mathrm{BR}\left(B \rightarrow X_{s} \ell^{+} \ell^{-}\right) \exp =(1.60 \pm 0.51) \times 10^{-6} .
$$

Concerning the forward-backward asymmetry $A_{\mathrm{FB}}$, the only existing positive data come from Belle [71, not yet precise enough to be conclusive on the presence of the zero.

For our forthcoming discussion, it will be useful to collect the following general properties of $B \rightarrow X_{s} \ell^{+} \ell^{-}$in the DR model that will be refined later on:

\footnotetext{
${ }^{6}$ SUSY contributions to $C_{9}$ and $C_{10}$ 64, 65, are completely negligible in the DR model.
} 
- The Wilson coefficients $C_{9}$ and $C_{10}$ receive only small NP contributions so that the departures of $B \rightarrow X_{s} \ell^{+} \ell^{-}, A_{\mathrm{FB}}$ and $\hat{s}_{0}$ from their SM values are governed by the modifications of $C_{7}$.

- Recalling that $C_{9}^{\mathrm{SM}}$ and $C_{7}^{\mathrm{SM}}$ have opposite sign, we observe that the flip of the sign of $C_{7}$ by NP contributions will strongly enhance $\operatorname{BR}\left(B \rightarrow X_{s} \ell^{+} \ell^{-}\right)$, while the enhancement of $\left|C_{7}\right|$ without the flip of its sign will suppress this branching ratio relative to the $\mathrm{SM}$ value 57,72

$$
\mathrm{BR}\left(B \rightarrow X_{s} \ell^{+} \ell^{-}\right)_{\mathrm{SM}}=(1.58 \pm 0.10) \times 10^{-6},
$$

that is in perfect agreement with experiment.

- The value of $\hat{s}_{0}$ is correlated with $\mathrm{BR}\left(B \rightarrow X_{s} \gamma\right)$ if $C_{7}$ has the SM sign. It increases with increasing $\mathrm{BR}\left(B \rightarrow X_{s} \gamma\right)$. This is a direct consequence of small NP contributions to $C_{9}$ in most NP models as pointed out in 73 .

- There is no zero in $A_{\mathrm{FB}}$ for $\operatorname{sign}\left(C_{7}\right)=-\operatorname{sign}\left(C_{7}^{\mathrm{SM}}\right)$.

\section{$5.5 \mathrm{~B}^{+} \rightarrow \tau^{+} \nu$}

Finally, we consider the tree-level decay $B^{+} \rightarrow \tau^{+} \nu$. In the SM, its branching ratio is simply given as follows,

$$
\mathrm{BR}\left(B^{+} \rightarrow \tau^{+} \nu\right)_{\mathrm{SM}}=\frac{G_{F}^{2} m_{B^{+}} M_{\tau}^{2}}{8 \pi}\left(1-\frac{M_{\tau}^{2}}{m_{B^{+}}^{2}}\right)^{2} F_{B^{+}}^{2}\left|V_{u b}\right|^{2} \tau_{B^{+}} .
$$

As the decay constant $F_{B^{+}} \approx F_{B_{d}}$ has still sizeable uncertainties, we consider instead the following ratios [74],

$$
\begin{aligned}
& \frac{\mathrm{BR}\left(B^{+} \rightarrow \tau^{+} \nu\right)_{\mathrm{SM}}}{\tau_{B^{+}}\left(\Delta M_{d}\right)_{\mathrm{SM}}}=\frac{3 \pi}{4 \eta_{B} S_{0}\left(\bar{m}_{t}\right) \hat{B}_{B_{d}}} \frac{M_{\tau}^{2}}{M_{W}^{2}}\left(1-\frac{M_{\tau}^{2}}{m_{B^{+}}^{2}}\right)^{2}\left|\frac{V_{u b}}{V_{t d}}\right|^{2}, \\
& \frac{\mathrm{BR}\left(B^{+} \rightarrow \tau^{+} \nu\right)_{\mathrm{SM}}}{\tau_{B^{+}}\left(\Delta M_{s}\right)_{\mathrm{SM}}}=\frac{3 \pi}{4 \eta_{B} S_{0}\left(\bar{m}_{t}\right) \hat{B}_{B_{d}}} \frac{M_{\tau}^{2}}{M_{W}^{2}} \frac{1}{\xi^{2}} \frac{m_{B^{+}}}{m_{B_{s}}}\left(1-\frac{M_{\tau}^{2}}{m_{B^{+}}^{2}}\right)^{2}\left|\frac{V_{u b}}{V_{c b}}\right|^{2},
\end{aligned}
$$

where we used $F_{B_{d}} \approx F_{B^{+}}, m_{B_{d}} \approx m_{B^{+}},\left|V_{t s}\right| \approx\left|V_{c b}\right|$ and the ratio $\xi$ defined as

$$
\xi=\frac{F_{B_{s}} \sqrt{\hat{B}_{B_{s}}}}{F_{B_{d}} \sqrt{\hat{B}_{B_{d}}}}
$$

The uncertainties on the right-hand side of (5.27) and (5.28) are comparable. In (5.27), the only hadronic uncertainty resides in $\hat{B}_{B_{d}}$, while in (5.28) there is an additional uncertainty in $\xi$. On the other hand, the ratio $\left|V_{u b} / V_{c b}\right|^{2}$ can be determined from tree-level decays without NP pollution, while $\left|V_{t d}\right|^{2}$ can clearly be affected by NP contributions.

Using the input parameters of Table 2 and 3, we find from (5.27)

$$
\mathrm{BR}\left(B^{+} \rightarrow \tau^{+} \nu\right)_{\mathrm{SM}}= \begin{cases}(0.87 \pm 0.11) \times 10^{-4}, & \left|V_{u b}\right|_{\text {UTfit }}, \\ (1.31 \pm 0.23) \times 10^{-4}, & \left|V_{u b}\right|_{\text {incl }},\end{cases}
$$




\begin{tabular}{|c|c|c|c|c|c|}
\hline Parameter & Value & Ref. & Parameter & Value & Ref. \\
\hline 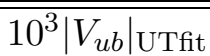 & $3.66(15)$ & 40 & 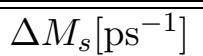 & 17.77(12) & {$[75]$} \\
\hline $10^{3}\left|V_{u b}\right|_{\text {incl }}$ & $4.49(33)$ & 775] & $\Delta M_{d}\left[\mathrm{ps}^{-1}\right]$ & $0.507(5)$ & 75 \\
\hline $10^{3}\left|V_{t d}\right|$ & $8.49(28)$ & 40] & $\bar{m}_{t}$ & $161.7(2.0)$ & \\
\hline$\hat{B}_{B_{d}}$ & $1.28(9)$ & & $m_{B_{s}}$ & $5.3661(6)$ & 76 \\
\hline$\xi$ & $1.23(6)$ & 777 & $m_{B^{+}}$ & $5.27913(31)$ & [76] \\
\hline$\eta_{B}$ & 0.55 & & $\tau_{B^{+}}\left[10^{-12} \mathrm{~s}\right]$ & $1.638(11)$ & 76 \\
\hline
\end{tabular}

Table 2: Input parameters for the SM prediction of $\mathrm{BR}\left(B^{+} \rightarrow \tau^{+} \nu\right)$. Dimensionful quantities are expressed in $\mathrm{GeV}$, unless otherwise specified.

where the first estimate uses the value for $\left|V_{u b}\right|$ resulting from the UTfit analysis of [40], while $\left|V_{u b}\right|_{\text {incl }}$ is the value resulting from inclusive decays alone. The corresponding values from (5.28) read

$$
\mathrm{BR}\left(B^{+} \rightarrow \tau^{+} \nu\right)_{\mathrm{SM}}= \begin{cases}(0.82 \pm 0.12) \times 10^{-4}, & \left|V_{u b}\right|_{\text {UTfit }}, \\ (1.24 \pm 0.24) \times 10^{-4}, & \left|V_{u b}\right|_{\text {incl }},\end{cases}
$$

showing that the formulae (5.27) and (5.28) give similar results.

We observe that the theoretical branching ratio with $\left|V_{u b}\right|_{\text {incl }}$ is closer to the experimental average between the Belle [78] and BaBar [79] results, which reads [80]

$$
\operatorname{BR}\left(B^{+} \rightarrow \tau^{+} \nu\right)_{\exp }=(1.31 \pm 0.48) \times 10^{-4},
$$

but the large experimental error precludes any clear cut conclusions at present. Yet, similarly to the case of the $B \rightarrow X_{s} \gamma$ decay, extensions of the SM that predict $\operatorname{BR}\left(B^{+} \rightarrow \tau^{+} \nu\right)$ to be smaller than the SM value seem to be disfavoured at present.

In this respect two-Higgs-doublet models of type-II, like the MSSM, where each doublet couples separately to up- and down-type quarks, are interesting as the interference between $W$ and $H^{+}$amplitudes is necessarily destructive [81]. One finds then [82, 83]

$$
R_{B \tau \nu}=\frac{\mathrm{BR}\left(B^{+} \rightarrow \tau^{+} \nu\right)^{\mathrm{DR}}}{\mathrm{BR}\left(B^{+} \rightarrow \tau^{+} \nu\right)^{\mathrm{SM}}}=\left[1-\frac{m_{B^{+}}^{2}}{m_{H^{+}}^{2}} \frac{\tan ^{2} \beta}{1+\epsilon_{0} \tan \beta}\right]^{2}\left|\frac{V_{u b}^{\mathrm{DR}}}{V_{u b}^{\mathrm{SM}}}\right|^{2} .
$$

We have explicitly shown the dependence on $V_{u b}$ since, in the DR model, the value of $\left|V_{u b}\right|$ turns out to be even smaller than $\left|V_{u b}\right|$ extracted from the UT SM fit.

\section{$5.6(g-2)_{\mu}$}

In principle we should also consider $(g-2)_{\mu}$, where the data seem to be above the SM expectations by roughly $3 \sigma$. In many supersymmetric models one finds, for large $\tan \beta$, $\mu>0$ and slepton masses $\mathrm{O}(400 \mathrm{GeV})$, additional contributions to $(g-2)_{\mu}$ that allow to fit the data. However, in the DR model, the slepton masses are larger than $1 \mathrm{TeV}$ and the NP contribution amounts to at most one $\sigma$ of the SM value. Therefore the DR model cannot fit the present data on $(g-2)_{\mu}$ and we will not include this observable in the global fit, keeping also in mind that the theoretical status of $(g-2)_{\mu}$ is not yet fully satisfactory [84, 85, 86]. 


\section{General picture}

Having the formulae for the FC observables at hand, we can discuss first the general pattern of these observables within the DR model. A detailed numerical analysis will be presented in the next section.

\section{Step 1}

In the DR model, due to the unification of Yukawa couplings, $\tan \beta$ is forced to be close to 50. This fact, as already stressed in section 5, requires $M_{A}$ to be sufficiently large in order for the predicted branching ratio of $B_{s} \rightarrow \mu^{+} \mu^{-}$in (5.4) to be consistent with the upper bound in (5.3). Typically we find $M_{A}>450 \mathrm{GeV}$. For such large Higgs masses, one has $M_{H^{+}} \approx M_{A} \approx M_{H_{0}}$ and this bound is also approximately valid for $M_{H^{+}}$and $M_{H_{0}}$.

\section{Step 2}

As already stressed in section 5.3, the large values of $M_{H^{+}}$and $M_{A}$ imply that NP contributions to $\operatorname{BR}\left(B \rightarrow X_{s} \gamma\right)$ are dominated by charginos, with the positive charged and neutral Higgs contributions (as well as those from gluinos and neutralinos) being subleading.

With $\mu>0$ it is possible to fit the data on $\operatorname{BR}\left(B \rightarrow X_{s} \gamma\right)$ by making the chargino contribution so large that $C_{7}^{\mathrm{SUSY}} \simeq-2 C_{7}^{\mathrm{SM}}$ at the $\mu_{b}$ scale. There are several problems with this choice.

First, there is the problem already stressed at the end of section 5.3. The possibility of having $C_{7}^{\mathrm{SUSY}} \simeq-2 C_{7}^{\mathrm{SM}}$ at the $\mu_{b}$ scale implies that the SUSY contribution is not quite a correction to the SM matching condition, but rather the opposite. With such a large correction coming from NP, the usual argument of neglecting NLO QCD corrections to NP contributions becomes invalid. One would need a control on the NP side at least as good as the one present in the SM contribution. This holds not only for the anomalous dimension matrix [87, but also for the matching conditions of the SUSY contributions [88, 48, 89, 90]. Indeed, with the use of only LO matching conditions for SUSY, we find a large sensitivity of the finely-tuned condition $C_{7}^{\mathrm{SUSY}} \simeq-2 C_{7}^{\mathrm{SM}}$ to the choice of the matching scale for the SUSY contributions. Such sensitivity becomes even stronger on the resulting $\mathrm{BR}\left(B \rightarrow X_{s} \gamma\right)$ and this makes a meaningful inclusion of the $\operatorname{BR}\left(B \rightarrow X_{s} \gamma\right)$ constraint in the numerical analysis practically impossible.

Second, there is no zero in the forward-backward asymmetry $A_{\mathrm{FB}}$, which is however still elusive experimentally [71].

Third and foremost, it has been shown in 91] that this solution for $C_{7}$ is actually excluded by the experimental data on $\operatorname{BR}\left(B \rightarrow X_{s} \ell^{+} \ell^{-}\right)$, provided the new physics contributions to the Wilson coefficients $\tilde{C}_{9}^{\text {eff }}$ and $\tilde{C}_{10}$ are small. In fact, the maximal ranges of such contributions in the MSSM with MFV found in [65] are too small to bring the theory prediction for $\operatorname{BR}\left(B \rightarrow X_{s} \ell^{+} \ell^{-}\right)$in accordance with the experimental data. These findings have also been confirmed in 72 .

In summary, we want to stress that although we cannot meaningfully take into account the case $C_{7}^{\mathrm{SUSY}} \simeq-2 C_{7}^{\mathrm{SM}}$ in the numerical analysis due to the theoretical uncertainties described above, we can exclude this case and impose $\operatorname{sign}\left(C_{7}\right) \equiv \operatorname{sign}\left(C_{7}^{\mathrm{SM}}\right)$ as a constraint in 
the global fitting procedure, because of the model-independent arguments brought forward in the previous paragraph, which are unaffected by such uncertainties.

\section{Step 3}

If $\mu$ is chosen to be positive and one attempts to be consistent with the data on $B \rightarrow X_{s} \ell^{+} \ell^{-}$ in (5.24) by keeping $C_{7}$ to have the SM sign, as discussed in the previous step, the negative contribution of charginos tends to suppress $\operatorname{BR}\left(B \rightarrow X_{s} \gamma\right)$ below acceptable values and can only be tamed by raising the squark masses until these contributions decouple.

\section{Step 4}

We next move to $\mu<0$. In this case, $C_{7}$ has the same sign as $C_{7}^{\mathrm{SM}}$, but due to constructive interference between SM, scalar and chargino contributions, it tends to be too large, unless squarks are sufficiently heavy. In this respect, we note that, for every given $m_{16}$, this case is not simply a reflection of the corresponding case with $\mu>0$. As we will see in the numerical section below, for negative $\mu$ the lightest squark masses are generically higher $(\gtrsim 2 \mathrm{TeV})$ than in the corresponding positive $\mu$ case.

\section{Step 5}

Let us finally look at $\mathrm{BR}\left(B^{+} \rightarrow \tau^{+} \nu\right)$. We have seen that in order to bring the SM value for this branching ratio close to its central experimental value, it was necessary to choose the tree-level value for $\left|V_{u b}\right|>4 \times 10^{-3}$. In the $\mathrm{DR}$ model, we do not have this freedom as $\left|V_{u b}\right|$ is in principle a prediction. In practice it is an outcome of the global fit to the model parameters and such fit definitely prefers a low value for $\left|V_{u b}\right|$, around $3.2 \times 10^{-3}$. With such value and the negative contribution from charged Higgses, we find typically

$$
\mathrm{BR}\left(B^{+} \rightarrow \tau^{+} \nu\right) \leq 0.6 \times 10^{-4}
$$

While this is not yet excluded, in view of the large experimental error in (5.32), also this decay could turn out to be problematic for the DR model if the central experimental value will remain above $1.0 \times 10^{-4}$ and the error will decrease by a factor of two.

\section{Final remarks}

In summary, we have shown that while it is possible through choice of the parameters to obtain the agreement of the DR model with a given single observable discussed above, simultaneous agreement for all observables is possible at most with very heavy sfermions. In the next section, we will present a detailed numerical analysis of these findings.

\section{Numerical analysis}

\subsection{Fitting procedure}

We now turn to describe the numerical strategy adopted to test the DR model. As we have seen in Section 3.1, the model is completely specified in terms of 24 parameters, listed in Table 1 and here collectively indicated as $\vec{\vartheta}$. After fixing them, it is possible to 
reconstruct the whole MSSM at low-energy scales, with a well-controlled theoretical error. The procedure, based on RGEs, which one adopts to connect the GUT scale model to low energies, has been described in Sections 3.2 and 4 .

Once the low-energy MSSM is specified, the model is testable. To this end, one needs a suitable set of observables, whose experimental determinations $\mathcal{O}_{i}$ should be as precise as possible and, on the theoretical side, calculable within the MSSM with sufficient accuracy. Since the MSSM is the low-energy result of the GUT scale model, the theoretical prediction for the observable $\mathcal{O}_{i}$ will be functions $f_{i}[\vec{\vartheta}]$ of the model parameters. In order to compare theory predictions with experimental values, one defines a suitable $\chi^{2}$-function as

$$
\chi^{2}[\vec{\vartheta}] \equiv \sum_{i=1}^{N_{\text {obs }}}\left(\frac{f_{i}[\vec{\vartheta}]-\mathcal{O}_{i}}{\sigma_{i}}\right)^{2},
$$

where the uncertainty $\sigma_{i}$ associated with the $i^{\text {th }}$ observable is defined as

$$
\sigma_{i}=\sqrt{\left(\sigma_{i}^{2}\right)_{\exp }+\left(\sigma_{i}^{2}\right)_{\text {theo }}} .
$$

Here $\left(\sigma_{i}\right)_{\exp }$ is the experimental RMS error and $\left(\sigma_{i}\right)_{\text {theo }}$ an estimate of the theoretical error associated with the $f_{i}[\vec{\vartheta}]$ calculation.

The $\chi^{2}$-function (7.1) is then minimized upon variation of the model parameters $\vec{\vartheta}$. To this end, we have adopted the minimization algorithm MIGRAD, which is part of the CERNlib library [92. The minimum value for the $\chi^{2}$-function provides then a quantitative test of the performance of the model in reproducing the observables entering the fit. We mention here that, strictly speaking, such test cannot be attached a statistically rigorous meaning, i.e. it is not a 'Pearson's test', since, e.g., the $\chi^{2}$-entries are not all independently measured observables. Nonetheless, the numerical value of the minimum for the function (7.1), as well as the single pulls in its entries, provide a good quantitative indication of the detailed performance of the model for the single observables.

The observables used in the fit are reported in Tables 35. Concerning the latter, the following comments are in order.

- The observables in Table 3 were already used - among the others - in the previous studies [1, 2] of the DR model, where the very good performance of the model in fitting them was demonstrated. We mention that the experimental determination of the observables themselves should not rely on any theoretical assumption which would be invalidated in the presence of NP, i.e. one should choose observables whose determination is NP-independent. This comment applies in particular to CKMrelated quantities, among which one keeps only those measured through tree-level processes and $\sin 2 \beta_{\psi K_{S}}$, which gives direct access to $\sin 2 \beta$ since the DR model has only one $\mathrm{CP}$ phase in the quark sector.

- The observables in Table 1 , on the other hand, represent the real novelty of our study with respect to the previous ones. Such FC processes are not calculated after the fitting procedure, but instead introduced directly in the $\chi^{2}$-function. The procedure to calculate these FC observables has been detailed in Section 5 . 


\begin{tabular}{|c|c|c|c|c|c|}
\hline Observable & Value $\left(\sigma_{\exp }\right)$ & Ref. & Observable & Value $\left(\sigma_{\exp }\right)$ & Ref. \\
\hline$M_{W}$ & $80.403(29)$ & 776 & $M_{\tau}$ & $\overline{1.777(0)}$ & 776 \\
\hline$M_{Z}$ & $91.1876(21)$ & 76 & $M_{\mu}$ & $0.10566(0)$ & 76 \\
\hline $10^{5} G_{\mu}$ & $1.16637(1)$ & 76 & $10^{3} M_{e}$ & $0.511(0)$ & 76 \\
\hline $1 / \alpha_{\mathrm{em}}$ & 137.036 & 76 & $\left|V_{u s}\right|$ & $0.2258(14)$ & 40 \\
\hline$\alpha_{s}\left(M_{Z}\right)$ & $0.1176(20)$ & 76 & $10^{3}\left|V_{u b}\right|$ & $4.1(0.4)$ & 41 \\
\hline$M_{t}$ & $170.9(1.8)$ & 76 & $10^{2}\left|V_{c b}\right|$ & 4.1 & $\sqrt{7}$ \\
\hline$m_{b}\left(m_{b}\right)$ & 4.20 & 76 & $\sin 2 \beta$ & 0.67 & $7 \overline{75}$ \\
\hline$m_{c}\left(m_{c}\right)$ & $1.25(9)$ & 76 & $10^{3} \Delta m_{31}^{2}\left[\mathrm{eV}^{2}\right]$ & $2.6(0.2)$ & 93 \\
\hline$m_{s}(2 \mathrm{GeV})$ & $0.095(25)$ & 76 & $10^{5} \Delta m_{21}^{2}\left[\mathrm{eV}^{2}\right]$ & $7.90(0.28)$ & 93 \\
\hline$m_{d}(2 \mathrm{GeV})$ & $0.005(2)$ & 76 & $\sin ^{2} 2 \theta_{12}$ & $0.852(32)$ & 93 \\
\hline$m_{u}(2 \mathrm{GeV})$ & $0.00225(75)$ & 76 & $\sin ^{2} 2 \theta_{23}$ & $0.996(18)$ & 93 \\
\hline
\end{tabular}

Table 3: Flavour conserving observables used in the fit. Dimensionful quantities are expressed in $\mathrm{GeV}$, unless otherwise specified.

\begin{tabular}{|c|c|c|}
\hline Observable & Value $\left(\sigma_{\exp }\right)\left(\sigma_{\text {theo }}\right)$ & Ref. \\
\hline $10^{3} \epsilon_{K}$ & $2.229(10)(252)$ & 76 \\
\hline$\Delta M_{s} / \Delta M_{d}$ & $35.0(0.4)(3.6)$ & {$[75,40$} \\
\hline $10^{4} \mathrm{BR}\left(B \rightarrow X_{s} \gamma\right)$ & $3.55(26)(46)$ & 46 \\
\hline $10^{6} \mathrm{BR}\left(B \rightarrow X_{s} \ell^{+} \ell^{-}\right), \quad q_{\ell^{+} \ell^{-}}^{2} \in[1,6] \mathrm{GeV}^{2}$ & $1.60(51)(40)$ & 72 \\
\hline $10^{4} \mathrm{BR}\left(B^{+} \rightarrow \tau^{+} \nu\right)$ & $1.31(48)(9)$ & 80 \\
\hline $\mathrm{BR}\left(B_{s} \rightarrow \mu^{+} \mu^{-}\right)$ & $<1.0 \times 10^{-7}$ & 32 \\
\hline
\end{tabular}

Table 4: FC observables used in the fit.

- In addition, we included in the fitting function a number of constraints, i.e. those on the lightest Higgs mass and on the lightest components of the SUSY spectrum, Table 5, and the constraint on the $\operatorname{BR}\left(B_{s} \rightarrow \mu^{+} \mu^{-}\right)$, Table 1 . These constraints are in the form of suitably smoothened step functions, which are added to the $\chi^{2}$-function of eq. (7.1). If any of the constraints is violated, the step functions add a large positive number to the $\chi^{2}$, while for respected constraints the returned value is zero, so that the $\chi^{2}$ is set back to its 'unbiased' definition (7.1).

Further comments on the determination of the theoretical errors are in order. First, one can note that among the observables in Table 3, some have a negligible experimental error. In this case, we took as overall uncertainly $0.5 \%$ of the experimental value, which we consider a realistic estimate of the numerical error associated with the calculations ${ }^{7}$. Concerning the theoret-

\begin{tabular}{|lcr|}
\hline Observable & Lower Bound & Ref. \\
\hline \hline$M_{h_{0}}$ & $114.4 \mathrm{GeV}$ & 76 \\
$m_{\tilde{t}}$ & $60 \mathrm{GeV}$ & 76 \\
$m_{\tilde{\chi}^{+}}$ & $104 \mathrm{GeV}$ & 76 \\
$m_{\tilde{g}}$ & $195 \mathrm{GeV}$ & 76 \\
\hline \hline
\end{tabular}

Table 5: Mass bounds used in the fit. ical errors on the flavour observables (Table 4), we note the following: the error on $\epsilon_{K}$ is

\footnotetext{
${ }^{7}$ Note that this error is more conservative than the $0.1 \%$ used in 1 , 2 . 2 .
} 


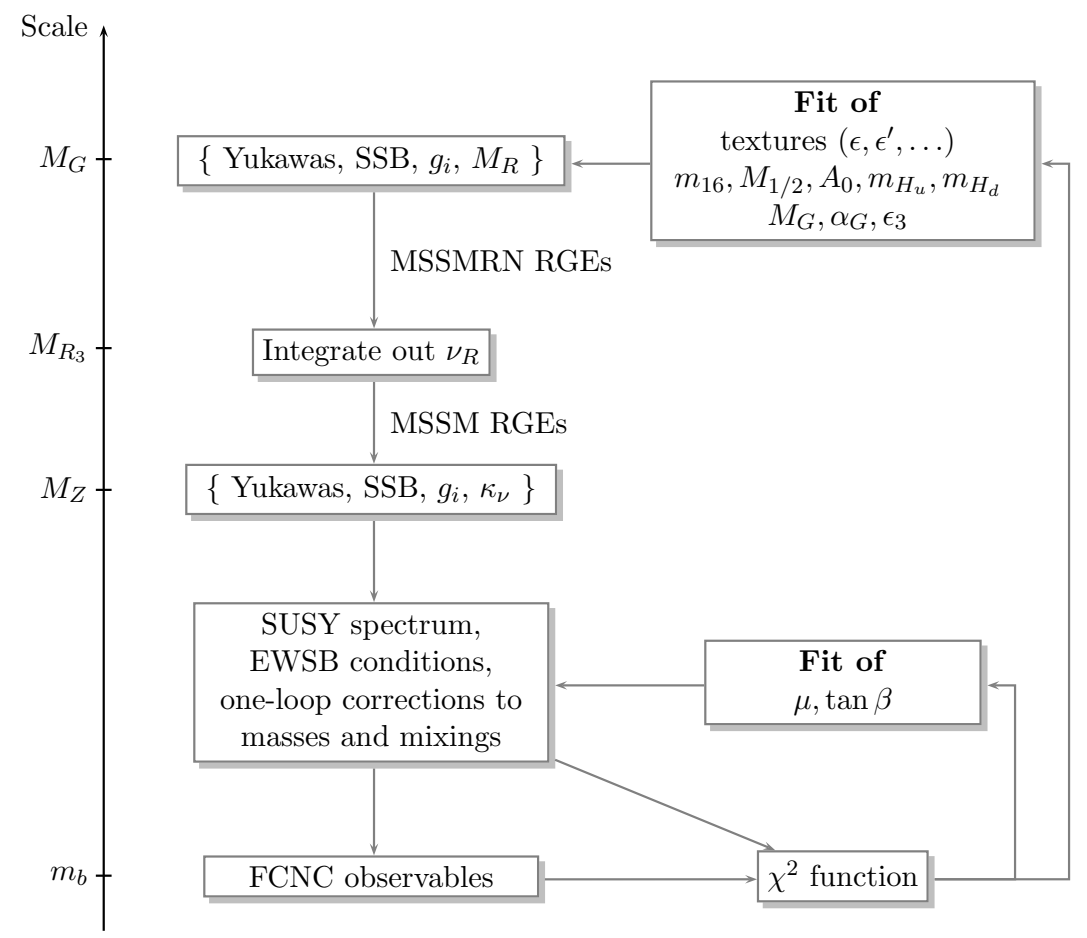

Figure 1: Schematic chart of the strategy followed in the fitting procedure.

basically that on the lattice parameter $\hat{B}_{K}$; the error on $\Delta M_{s} / \Delta M_{d}$ keeps into account that on the SM contribution, dominated by $\xi^{2}$ and that on the NP contributions, dominated by the scalar $P_{L} \otimes P_{R}$ operators; the error on $\mathrm{BR}\left(B^{+} \rightarrow \tau^{+} \nu\right)$, after normalization as in eq. (5.27), is only that on $\hat{B}_{d}$; the error on $\operatorname{BR}\left(B \rightarrow X_{s} \gamma\right)$ is taken as twice the total theoretical error associated with the $\mathrm{SM}$ calculation [46]; finally the error on $\mathrm{BR}\left(B \rightarrow X_{s} \ell^{+} \ell^{-}\right)$is taken as $25 \%$ of the experimental result, and is estimated from the spread of the theoretical predictions after variations of the scale of matching of the SUSY contributions.

We next turn to the generic strategy adopted to minimize the $\chi^{2}$-function with respect to the parameters $\vec{\vartheta}$. We note that, among them, $m_{H_{u, d}}, \mu$ and $\tan \beta$ are those responsible for EW symmetry breaking, and the $\chi^{2}$-function manifests a particularly sensitive dependence on them, especially on $m_{H_{u, d}}$. As a consequence, such parameters are varied first (keeping the other fixed to initial guesses), in order to successfully find an EW symmetry breaking minimum, thereafter varying the rest of the parameters. This procedure is schematically described in the flow-chart of Fig. 1. As a final step, all the parameters are varied simultaneously.

Among the model parameters, the ones in the SUSY sector are particularly interesting since they set the scale of the SUSY particles' masses. This is especially true for $m_{16}$ and $\mu$. All the other parameters can be left free in the fit, since their typically allowed range of variation is quite narrow. In particular, when $m_{16}$ is fixed and $\mu$ is positive, the fit prefers regions of the remaining parameter space such that

$$
\mu, M_{1 / 2} \ll m_{16}, \quad-A_{0} \simeq 2 m_{16},
$$


which is favoured by third generation Yukawa unification [94, 95, 96. Concerning the first of relations (7.3),$M_{1 / 2}$ is bounded from above because otherwise the bottom mass is pushed up beyond acceptable values by large gluino corrections. In fact, we find that $M_{1 / 2}$ is most of the times chosen in the range $[140,400] \mathrm{GeV}$, where the lower bound results from the chargino mass bound in Table 5 .

The second of relations (7.3) leads to an inverted scalar mass hierarchy [97], i.e. heavy first and second generation sfermions, but lighter third generation sfermions. For the values of $m_{16}$ considered here, namely $m_{16} \geq 4 \mathrm{TeV}$, and for $\mu>0$, this condition also helps to obtain the correct prediction for $m_{b}$ [94, 95].

On the other hand, the allowed interval for $\mu$ is generically wider, for every fixed value of $m_{16}$. As a consequence, our main strategy is to study the model behavior for different choices of $\left\{m_{16}, \mu\right\}$, and for each of them, let the rest of the parameter space free to be determined by the minimization procedure. In the next section we now turn to describe the various scenarios considered in the $\left\{m_{16}, \mu\right\}$ plane.

\subsection{Scenarios}

We considered increasing values of $m_{16}$ starting from $4 \mathrm{TeV}$, which represents the "minimum' value for successful fits to the observables of Table 3 [1, 2]. For each fixed value of $m_{16}$ we then studied the $\mu$ dependence by performing fits with different initial guesses for this parameter. All input values for the model parameters corresponding to these scenarios are listed in Table 6. In the following, we describe in detail our findings.

\subsection{1 $m_{16}=4 \mathrm{TeV}, \mu>0$}

Given the inverted scalar mass hierarchy, the relatively low value of $m_{16}$ leads to stop masses below $1 \mathrm{TeV}$, resulting in a large chargino contribution to $\operatorname{BR}\left(B \rightarrow X_{s} \gamma\right)$.

As a matter of fact, the preferred region of NP contributions to the latter decay mode reverses the sign for $C_{7}\left(\mu_{b}\right): C_{7}\left(\mu_{b}\right) \simeq-C_{7}^{\mathrm{SM}}\left(\mu_{b}\right)$. On the fine-tuned character of this case we have already commented in Sections 5.3 and 6. We stress again that within the DR model, this solution is not viable in view of the implied enhancement to the branching ratio of $B \rightarrow X_{s} \ell^{+} \ell^{-}$. Model-independent analyses [91, 72] show in fact that to compensate for such enhancement, one would need substantial contributions to the Wilson coefficients $\tilde{C}_{9}^{\text {eff }}$ and $\tilde{C}_{10}$ of the operators in eq. (5.18). However, within the DR model, these Wilson coefficients are always SM-like to an excellent approximation.

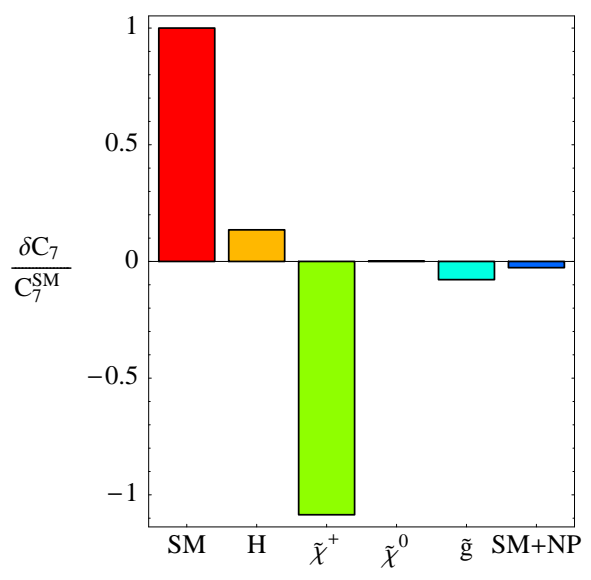

Figure 2: Contributions to $C_{7}\left(\mu_{b}\right)$ for the scenario of section 7.2.1.

As a consequence of the above, we have studied the viability of having the SM sign in $C_{7}\left(\mu_{b}\right)$ by imposing this condition as a constraint on the $\chi^{2}$ function. The typical fit result in this case is illustrated in Table 7 . 
The fit displays the main problem of the model in this regime for $\left\{m_{16}, \mu\right\}$, i.e. a $5 \sigma$ discrepancy in the predicted $\operatorname{BR}\left(B \rightarrow X_{s} \gamma\right)$. Even imposing the SM sign on $C_{7}\left(\mu_{b}\right)$, the contribution from charginos is still too large in magnitude, and none of the other NP contributions is able to compensate for it. The situation is illustrated in Fig. 2.

A second, though less severe, problem is the predicted value for $\operatorname{BR}\left(B^{+} \rightarrow \tau^{+} \nu\right)$, which is roughly $2 \sigma$ too low with respect to the experimental average (5.32). This problem is strictly connected to the quite low value for $\left|V_{u b}\right| \approx 3.2 \times 10^{-3}$ predicted by the model. We found this feature to hold irrespective of the values chosen for $\left\{m_{16}, \mu\right\}$, so that it should be connected to the specific Yukawa textures of the model.

\subsection{2 $m_{16}=6 \mathrm{TeV}, \mu>0$}

Since the $\operatorname{BR}\left(B \rightarrow X_{s} \gamma\right)$ problem is related to the low value for $\mu$ required (at least for positive $\mu$ ) by $m_{16}=4 \mathrm{TeV}$, we have tried to increase the latter in order to understand how fastly decoupling is effective in mildening the problem.

For $m_{16}=6 \mathrm{TeV}$ and $\mu>0$ the preferred range for $\mu$ is roughly $\mu \in[800,1000] \mathrm{GeV}$. A typical fit result is displayed in Table 8, corresponding to the case $\mu=953 \mathrm{GeV}$. As a matter of fact, the discrepancy in $B \rightarrow X_{s} \gamma$ is tamed to roughly $2.3 \sigma$, since chargino contributions are less important than in the $m_{16}=4 \mathrm{TeV}$ cases. The various contributions to $C_{7}\left(\mu_{b}\right)$ for the fit in Table 8 are displayed in Fig. 3. By decreasing $\mu$ below $\approx 800 \mathrm{GeV}$, the prediction for $B \rightarrow X_{s} \gamma$ fastly worsens. For example, for a converged fit with $\mu=430 \mathrm{GeV}$, we found that the discrepancy is already at the $4.2 \sigma$ level. Concerning $B^{+} \rightarrow \tau^{+} \nu$, as anticipated above, the predicted

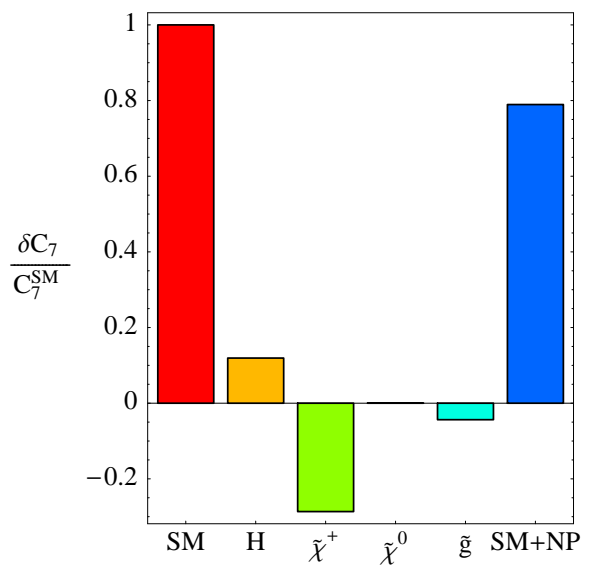

Figure 3: Contributions to $C_{7}\left(\mu_{b}\right)$ for the scenario of section 7.2.2.

rate remains always roughly $2 \sigma$ off.

As a final remark, while the case $m_{16}=6 \mathrm{TeV}$ allows, for suitable $\mu$, a smaller discrepancy in $B \rightarrow X_{s} \gamma$, the latter comes with the price of a much higher mass for the lightest up-type squark, as evident by comparing the corresponding values in Table 11 .

\subsection{3 $m_{16}=10 \mathrm{TeV}, \mu>0$}

Increasing $m_{16}$ further, the chargino contribution to $\mathrm{BR}\left(B \rightarrow X_{s} \gamma\right)$ becomes comparable in size to the charged Higgs contribution, resulting in acceptable values for this branching ratio. For example, in the fit of Table 9, the pull in this observable is reduced to $1.3 \sigma$. On the other hand, the pull resulting from $B^{+} \rightarrow \tau^{+} \nu$ is not significantly ameliorated compared to the previous cases. In addition, the lightest squark is as heavy as $1.9 \mathrm{TeV}$.

\subsection{4 $m_{16}=4 \mathrm{TeV}, \mu<0$}

We explored also the case with negative $\mu$. In this instance, relations in eq. (7.3) (with $\mu \rightarrow|\mu|)$ do not apparently need to be fulfilled. As a matter of fact, we found satisfactory 
fits for a quite wide range of $\mu: \mu \in-[2100,400]$ and $A_{0}$ is always lower in magnitude than the value required by the second relation in eq. (7.3), typically leading to very small $A_{t}$. As a consequence, the squark mass spectrum does not fulfill an inverted mass hierarchy [97, 96] and squark masses are generically very heavy. In this case, also heavy Higgses are found to have generically larger masses, $\gtrsim 1.5 \mathrm{TeV}$. A typical result is shown in Table 10 and the displayed features remain basically the same in the full allowed range for $\mu$.

We observe that, in this case, negative values of $\mu$, large squark masses and small values for $A_{t}$ imply small threshold corrections to $m_{b}$ and therefore allow to have successful Yukawa unification away from the inverted mass hierarchy condition. We have then investigated whether an acceptable fit away from inverted mass hierarchy could also be obtained for $\mu>0^{8}$. In the latter case, the most important contributions to the $m_{b}$ threshold correction have the same sign and consequently one generically needs larger squark masses than for $\mu<0$, in order to reproduce the right $m_{b}$ value. In fact we find that, unless $m_{16} \gtrsim 6 \mathrm{TeV}$, the prediction on $m_{b}$ is $4 \sigma$ too large ${ }^{9}$ and consequently fits with $\mu>0$ and away from the inverted mass hierarchy perform worse than the corresponding negative $\mu$ cases.

We also note that, in the $\left\{m_{16}, \mu\right\}$ mass scenario considered in the present subsection, the predicted value for $\mathrm{BR}\left(B \rightarrow X_{s} \gamma\right)$ is always larger than the SM prediction and close to the experimental value. In fact, small $A_{t}$ implies negligible chargino contributions, so that the main correction is the one from Higgses. However, since the lightest stop is around 2.6 TeV, this scenario clashes with the motivation for SUSY as a solution to the Higgs fine-tuning problem.

\subsection{Results}

Considering the discussion in the last section, it is apparent that for positive $\mu$, the tension between the three decays $B \rightarrow$ $X_{s} \gamma, B \rightarrow X_{s} \ell^{+} \ell^{-}$and $B_{s} \rightarrow \mu^{+} \mu^{-}$can be relieved by raising the universal sfermion mass $m_{16}$ beyond $8 \mathrm{TeV}$. This is demonstrated in Fig. 1 , showing the total $\chi^{2}$ and the contribution of $B \rightarrow X_{s} \gamma$ to the $\chi^{2}$ for increasing values of $m_{16}$. For negative $\mu$, fits with comparable $\chi^{2}$ can be achieved with lower values of $m_{16}$, but not with lighter squarks, as the lightest squark is still very heavy in these cases.

However, it is well-known that the supersymmetric solution to the gauge hierarchy problem requires light third generation sfermions. Therefore, light stops are favourable to reduce fine-tuning. To show

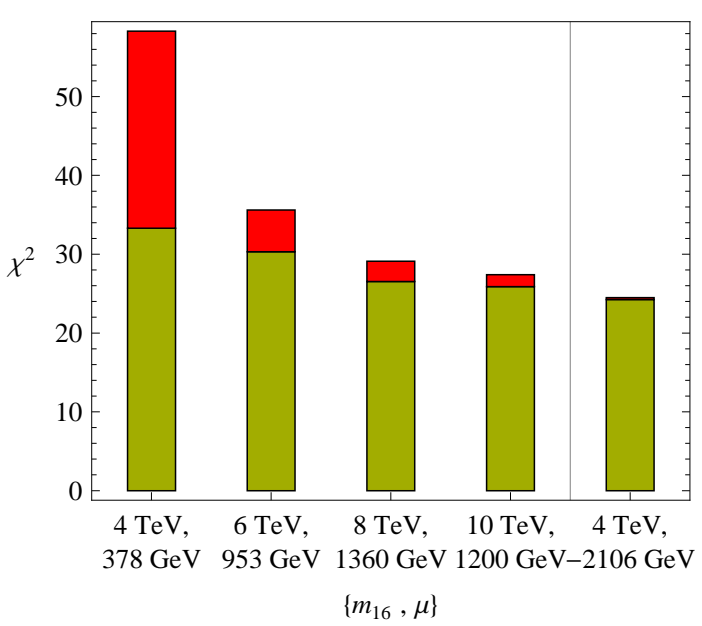

Figure 4: Total $\chi^{2}$ (whole bar) and contribution of $B \rightarrow X_{s} \gamma$ to the $\chi^{2}$ (red bar) for the best fits with positive $\mu$ and $m_{16}=4,6,8$ and $10 \mathrm{TeV}$, respectively, as well as the best fit with negative $\mu$. the amount of splitting between fermion and sfermion masses needed in the DR model, we plot the lightest stop mass $m_{\tilde{t}_{1}}$ versus the total $\chi^{2}$ for all fits with positive as well as neg-

\footnotetext{
${ }^{8}$ We warmly thank R. Dermíšek for drawing this point to our attention.

${ }^{9}$ One should also take into account the quite precisely known value for $m_{b}$ assumed in the present paper.
} 
ative $\mu$ we obtained (see Fig. 5). There is obviously a strong correlation between the stop mass and the quality of the fit, demonstrating that $m_{\tilde{t}_{1}}$ has to be at least as large as $1.8 \mathrm{TeV}$ to cure the problems with the three aforementioned decays. This is significantly heavier than the masses considered in [1], 2] and may be difficult to reconcile with naturalness.

In addition, a number of problems of the model persist even for very large $m_{16}$. These are the issues related to the Yukawa textures: the up-quark mass, $V_{u b}$ and $\sin 2 \beta$, and as a result also $\Delta M_{s} / \Delta M_{d}$ and $B^{+} \rightarrow$ $\tau^{+} \nu$. This is why there are no points with $\chi^{2} \lesssim 25$ in Fig. 5. The reason for the much higher $\chi^{2}$ contribution from flavour conserving quantities as compared to [1, 2] is mainly due to updated experimental values and reduced experimental errors, especially in $\left|V_{u s}\right|,\left|V_{u b}\right|$ and $m_{b}$.

For a given value of $m_{16}$, we found that successful fits could only be obtained in a limited range of allowed values for $\mu$. The preferred value of $\mu$ increased with increasing $m_{16}$, as can be seen for the best

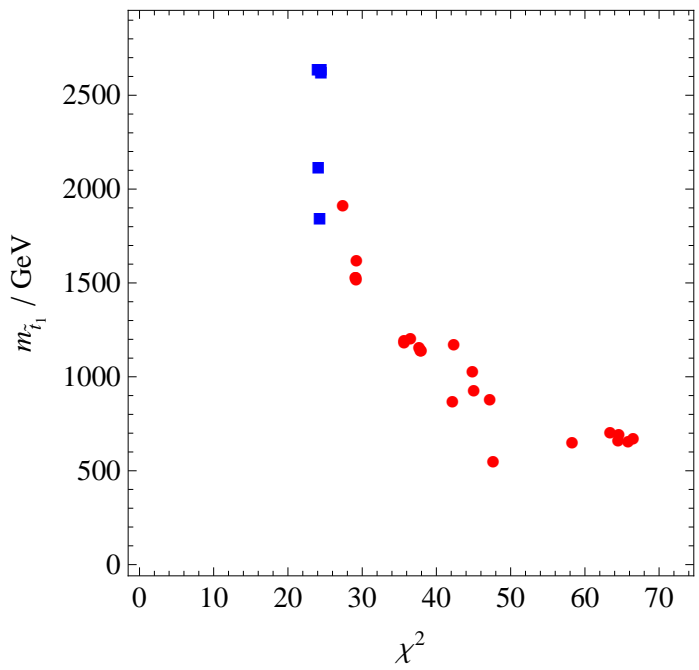

Figure 5: Total $\chi^{2}$ vs. the lightest stop mass for all obtained fits. Red circular points are fits with positive $\mu$, blue squares with negative $\mu$. fit points in Fig. 4 . This fact could give rise to an additional problem. Although we did not include the dark matter density as a constraint in our $\chi^{2}$ analysis, we conjecture that such large values of $\mu$ would give rise to a relic abundance of neutralinos incompatible with the WMAP measurements, for the following reason. Because of the extremely heavy sfermions, the dominant annihilation channel for the neutralino in this scenario is through an $s$-channel pseudoscalar Higgs. While this rate is already suppressed by the large $M_{A}$, the coupling of neutralinos to the pseudoscalar Higgs is additionally suppressed by large $\mu$. This would result in an overabundance of relic neutralinos incompatible with observations. Solving this problem by resonant neutralino annihilation with $m_{\tilde{\chi}^{0}} \approx M_{A} / 2$, as was advocated in an extensive analysis of dark matter in this class of models [98, 99], is not possible because of the large $M_{A}$ and the small $M_{1 / 2}$ preferred by the fit.

\section{Conclusions}

In this paper we have performed a detailed analysis of the SO(10) SUSY GUT model with $D_{3}$ family symmetry of Dermíšek and Raby [1, 2].

This model is entirely specified in terms of 24 parameters. Once they are fixed, the whole MSSM parameter space (including its SM subset) is predicted at low energies with the help of RGEs. The common dependence on the model parameters strongly correlates all the low energy observables, in contrast with the direct consideration of the MSSM at the EW scale, where the CKM parameters and the fermion masses are fully independent of 
the SUSY particle spectrum. We find that the DR model gives a satisfactory description of the quark and lepton matrices as well as of the PMNS and CKM mixing matrices with one exception: the CKM element $\left|V_{u b}\right|$ turns out to be significantly smaller than $\left|V_{u b}\right|_{\text {incl }}$ extracted from inclusive tree-level decays and even smaller than $\left|V_{u b}\right|_{\text {excl }}$. The above findings are mostly a confirmation of previous studies of the model.

The main novelty of our study, with respect to similar analyses of SUSY GUT models found in the literature, is that we analyze simultaneously the mass spectra of quark and leptons, the CKM and PMNS mixing matrices, the SUSY mass spectrum and its implied corrections to the FC processes $B_{s} \rightarrow \mu^{+} \mu^{-}, B \rightarrow X_{s} \gamma, B \rightarrow X_{s} \ell^{+} \ell^{-}, B^{+} \rightarrow \tau^{+} \nu$ and the

$B_{d, s}-\bar{B}_{d, s}$ mass differences $\Delta M_{d, s}$. The performance of the model is assessed by means of a global fit to the above mentioned observables.

The inclusion of the FC processes listed above turns out to be a crucial test of the mass hierarchies predicted by the model for the SUSY spectrum. In fact, such hierarchies unavoidably manifest themselves in loop corrections, and FC observables remain the most sensitive probes of such corrections. Our analysis demonstrates that the simultaneous description of all the FC processes listed above is a serious challenge for the DR model. In view of the specific way this failure is realized, we suspect that this is a problem of a wider class of SUSY GUTs in which the presence of Yukawa unification implies $\tan \beta \simeq 50$, unless non-minimal sources of flavour violation are introduced.

Our main message is the following one. To really assess the viability of models for flavour parameters, it is essential not only to verify their ability to reproduce quark and lepton mass spectra and mixing matrices - in itself an already notable achievement but also to test the consistency with the data on available FC processes, since the latter have a simultaneous sensitivity to mixing matrices and new particles' spectra. In the DR model example, FC processes are in fact the best probes available to the SUSY part of the spectrum, where information from direct detection is missing. It turns out that the DR model - otherwise successful for quark and lepton masses as well as for the CKM and PMNS matrices - is challenged only when specifically tested in the simultaneous description of quark FCNC processes. The failure in the description of these data makes the viability of the DR model questionable from the present perspective, but hopefully offers insights on further lines of development along similar classes of models.

\section{Acknowledgements}

We warmly acknowledge Radovan Dermíšek and Stuart Raby for useful discussions and for a critical reading of the manuscript. This work has been supported in part by the Cluster of Excellence "Origin and Structure of the Universe" and by the German Bundesministerium für Bildung und Forschung under contract 05HT6WOA. D.G. also warmly acknowledges the support of the A. von Humboldt Stiftung.

\section{Note added}

During the completion of the present work, a new bound on the branching ratio for $B_{s} \rightarrow$ 
$\mu^{+} \mu^{-}$has been presented at the HEP 2007 conference [100]. The latter results from a combined analysis of the CDF and D $\varnothing$ data and reads

$$
\mathrm{BR}\left(B_{s} \rightarrow \mu^{+} \mu^{-}\right) \mathrm{CDF+ \textrm {D } \emptyset}<5.8 \times 10^{-8}, \quad(95 \% \text { C.L. }) .
$$

This bound represents a considerable improvement over the one given in eq. (5.3).

In addition, at the SUSY 2007 conference it was presented a new (preliminary) result on the $\operatorname{BR}\left(B^{+} \rightarrow \tau^{+} \nu\right)$ from the BaBar collaboration [101], which reads

$$
\mathrm{BR}\left(B^{+} \rightarrow \tau^{+} \nu\right)_{\text {BaBar,prelim. }}=\left(1.2 \pm 0.4_{\text {stat }} \pm 0.3_{\mathrm{bkg}} \pm 0.2_{\mathrm{eff}}\right) \times 10^{-4}
$$

Performing the weighted average between the result in eq. (8.2) and the Belle result 78 one obtains

$$
\operatorname{BR}\left(B^{+} \rightarrow \tau^{+} \nu\right)_{\text {new }}=(1.41 \pm 0.43) \times 10^{-4}
$$

The new averages in eqs. (8.1) and (8.3) further strengthen our conclusions.

\section{References}

[1] R. Dermíšek and S. Raby, Bi-large neutrino mixing and CP violation in an SO(10) SUSY GUT for fermion masses, Phys. Lett. B622 (2005) 327-338, hep-ph/0507045].

[2] R. Dermíšek, M. Harada, and S. Raby, SO(10) SUSY GUT for fermion masses: Lepton flavor and CP violation, Phys. Rev. D74 (2006) 035011, hep-ph/0606055.

[3] R. Dermíšek and S. Raby, Fermion masses and neutrino oscillations in SO(10) SUSY GUT with $D(3) \times$ U(1) family symmetry, Phys. Rev. D62 (2000) 015007, hep-ph/9911275.

[4] C. D. Froggatt and H. B. Nielsen, Hierarchy of Quark Masses, Cabibbo Angles and CP Violation, Nucl. Phys. B147 (1979) 277.

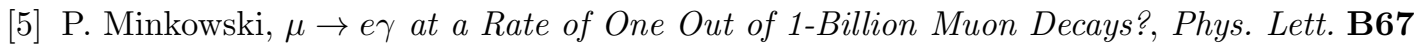
(1977) 421.

[6] M. Gell-Mann, P. Ramond and R. Slansky, Supergravity (P. van Nieuwenhuizen and D.Z. Freedman eds.), North-Holland, Amsterdam, 1979, p. 315; T. Yanagida, in Proceedings of the Workshop on the unified theory and the baryon number of the universe (O. Sawada and A. Sugamoto eds.), KEK report No. 79-18, Tsukuba, Japan, 1979, p. 95; S. L. Glashow, The future of elementary particle physics, in Proceedings of the 1979 Cargèse Summer Institute on Quarks and Leptons (M. Lévy et al. eds.), Plenum Press, New York, 1980, pp. 687-713.

[7] R. N. Mohapatra and G. Senjanovic, Neutrino mass and spontaneous parity nonconservation, Phys. Rev. Lett. 44 (1980) 912.

[8] P. Ramond, The family group in grand unified theories, hep-ph/9809459.

[9] R. Ruiz de Austri, R. Trotta, and L. Roszkowski, A Markov chain Monte Carlo analysis of the CMSSM, JHEP 05 (2006) 002, hep-ph/0602028.

[10] L. Roszkowski, R. Ruiz de Austri, and R. Trotta, On the detectability of the CMSSM light Higgs boson at the Tevatron, JHEP 04 (2007) 084, hep-ph/0611173. 
[11] L. Roszkowski, R. Ruiz de Austri, and R. Trotta, Implications for the constrained MSSM from a new prediction for $b \rightarrow s \gamma, 0705.2012$.

[12] J. R. Ellis, S. Heinemeyer, K. A. Olive, A. M. Weber, and G. Weiglein, The Supersymmetric Parameter Space in Light of B-physics Observables and Electroweak Precision Data, 0706.0652 .

[13] J. Hisano, T. Moroi, K. Tobe, and M. Yamaguchi, Lepton-Flavor Violation via Right-Handed Neutrino Yukawa Couplings in Supersymmetric Standard Model, Phys. Rev. D53 (1996) 2442-2459, hep-ph/9510309].

[14] S. Antusch, J. Kersten, M. Lindner, M. Ratz, and M. A. Schmidt, Running neutrino mass parameters in see-saw scenarios, JHEP 03 (2005) 024, hep-ph/0501272.

[15] S. T. Petcov, S. Profumo, Y. Takanishi, and C. E. Yaguna, Charged lepton flavor violating decays: Leading logarithmic approximation versus full RG results, Nucl. Phys. B676 (2004) 453-480, hep-ph/0306195.

[16] S. Antusch, J. Kersten, M. Lindner, and M. Ratz, Neutrino mass matrix running for non-degenerate see-saw scales, Phys. Lett. B538 (2002) 87-95, hep-ph/0203233].

[17] S. P. Martin and M. T. Vaughn, Two loop renormalization group equations for soft supersymmetry breaking couplings, Phys. Rev. D50 (1994) 2282, hep-ph/9311340.

[18] G. D'Ambrosio, G. F. Giudice, G. Isidori, and A. Strumia, Minimal flavour violation: An effective field theory approach, Nucl. Phys. B645 (2002) 155-187, hep-ph/0207036.

[19] J. Rosiek, Complete set of Feynman rules for the Minimal Supersymmetric extension of the Standard Model, Phys. Rev. D41 (1990) 3464. Erratum [hep-ph/9511250].

[20] D. M. Pierce, J. A. Bagger, K. T. Matchev, and R.-J. Zhang, Precision corrections in the Minimal Supersymmetric Standard Model, Nucl. Phys. B491 (1997) 3-67, hep-ph/9606211.

[21] P. H. Chankowski, S. Pokorski, and J. Rosiek, Complete on-shell renormalization scheme for the minimal supersymmetric Higgs sector, Nucl. Phys. B423 (1994) 437-496, hep-ph/9303309.

[22] S. Heinemeyer, W. Hollik, and G. Weiglein, FeynHiggs: A program for the calculation of the masses of the neutral CP-even Higgs bosons in the MSSM, Comput. Phys. Commun. 124 (2000) 76-89, hep-ph/9812320.

[23] S. Heinemeyer, W. Hollik, and G. Weiglein, The masses of the neutral CP-even Higgs bosons in the MSSM: Accurate analysis at the two-loop level, Eur. Phys. J. C9 (1999) 343-366, hep-ph/9812472.

[24] G. Degrassi, S. Heinemeyer, W. Hollik, P. Slavich, and G. Weiglein, Towards high-precision predictions for the MSSM Higgs sector, Eur. Phys. J. C28 (2003) 133-143, hep-ph/0212020.

[25] M. Frank et. al., The Higgs boson masses and mixings of the complex MSSM in the Feynman-diagrammatic approach, hep-ph/0611326.

[26] M. Carena, D. Garcia, U. Nierste, and C. E. M. Wagner, Effective Lagrangian for the $\bar{t} b H^{+}$ interaction in the MSSM and charged Higgs phenomenology, Nucl. Phys. B577 (2000) 88-120, hep-ph/9912516. 
[27] T. Blažek, S. Raby, and S. Pokorski, Finite supersymmetric threshold corrections to CKM matrix elements in the large $\tan \beta$ regime, Phys. Rev. D52 (1995) 4151-4158, hep-ph/9504364.

[28] A. J. Buras, P. H. Chankowski, J. Rosiek, and L. Slawianowska, $\Delta M_{d, s}, B_{d . s}^{0} \rightarrow \mu^{+} \mu^{-}$and $B \rightarrow X_{s} \gamma$ in supersymmetry at large $\tan (\beta)$, Nucl. Phys. B659 (2003) 3, hep-ph/0210145.

[29] P. H. Chankowski and P. Wasowicz, Low energy threshold corrections to neutrino masses and mixing angles, Eur. Phys. J. C23 (2002) 249-258, hep-ph/0110237.

[30] A. J. Buras, Relations between $\Delta M_{s, d}$ and $B_{s, d} \rightarrow \mu \bar{\mu}$ in models with minimal flavour violation, Phys. Lett. B566 (2003) 115-119, hep-ph/0303060].

[31] M. Blanke, A. J. Buras, D. Guadagnoli, and C. Tarantino, Minimal flavour violation waiting for precise measurements of $\Delta M_{s}, S_{\psi \phi}, A_{\mathrm{SL}}^{s},\left|V_{u b}\right|, \gamma$ and $B_{s, d}^{0} \rightarrow \mu^{+} \mu^{-}, J H E P 10$ (2006) 003, hep-ph/0604057.

[32] http://www-cdf.fnal.gov/physics/new/bottom/060316.blessed-bsmumu3 and CDF Public note 8176.

[33] S. R. Choudhury and N. Gaur, Dileptonic decay of $B_{s}$ meson in SUSY models with large $\tan (\beta)$, Phys. Lett. B451 (1999) 86-92, hep-ph/9810307.

[34] K. S. Babu and C. F. Kolda, Higgs-mediated $B_{0} \rightarrow \mu^{+} \mu^{-}$in minimal supersymmetry, Phys. Rev. Lett. 84 (2000) 228-231, hep-ph/9909476.

[35] G. Isidori and A. Retico, Scalar flavour-changing neutral currents in the large- $\tan (\beta)$ limit, JHEP 11 (2001) 001, hep-ph/0110121.

[36] M. Carena, A. Menon, and C. E. M. Wagner, Challenges for MSSM Higgs searches at Hadron Colliders, 0704.1143.

[37] D. Becirevic, V. Gimenez, G. Martinelli, M. Papinutto, and J. Reyes, B-parameters of the complete set of matrix elements of $\Delta B=2$ operators from the lattice, JHEP 04 (2002) 025, hep-lat/0110091.

[38] A. Freitas, E. Gasser, and U. Haisch, Supersymmetric large $\tan (\beta)$ corrections to $\Delta M_{d, s}$ and $B_{d, s} \rightarrow \mu^{+} \mu^{-}$revisited, hep-ph/0702267. See also M. Gorbahn, S. Jäger, U. Nierste and S. Trine. In preparation.

[39] CDF Collaboration, A. Abulencia et. al., Observation of $B_{s}^{0}-\bar{B}_{s}^{0}$ oscillations, Phys. Rev. Lett. 97 (2006) 242003, hep-ex/0609040.

[40] UTfit website: http://www.utfit.org.

[41] CKMfitter website: http://ckmfitter.in2p3.fr.

[42] A. J. Buras, P. H. Chankowski, J. Rosiek, and L. Slawianowska, Correlation between $\Delta M_{s}$ and $B_{s, d}^{0} \rightarrow \mu^{+} \mu^{-}$in supersymmetry at large $\tan (\beta)$, Phys. Lett. B546 (2002) 96-107, hep-ph/0207241.

[43] Belle Collaboration, P. Koppenburg et. al., An inclusive measurement of the photon energy spectrum in $b \rightarrow$ s $\gamma$ decays, Phys. Rev. Lett. 93 (2004) 061803, hep-ex/0403004.

[44] BaBar Collaboration, B. Aubert et. al., Measurement of the branching fraction and photon energy moments of $B \rightarrow X_{s} \gamma$ and $A_{\mathrm{CP}}\left(B \rightarrow X_{s+d} \gamma\right)$, Phys. Rev. Lett. 97 (2006) 171803, hep-ex/0607071. 
[45] Heavy Flavor Averaging Group (HFAG) Collaboration, E. Barberio et. al., Averages of b-hadron properties at the end of 2005, hep-ex/0603003.

[46] M. Misiak et. al., The first estimate of $\mathrm{B}\left(\bar{B} \rightarrow X_{s} \gamma\right)$ at $O\left(\alpha_{s}^{2}\right)$, Phys. Rev. Lett. 98 (2007) 022002, hep-ph/0609232.

[47] T. Becher and M. Neubert, Analysis of $B r\left(B \rightarrow X_{s} \gamma\right)$ at NNLO with a cut on photon energy, Phys. Rev. Lett. 98 (2007) 022003, hep-ph/0610067.

[48] C. Bobeth, M. Misiak, and J. Urban, Matching conditions for $b \rightarrow s \gamma$ and $b \rightarrow s$ gluon in extensions of the standard model, Nucl. Phys. B567 (2000) 153-185, hep-ph/9904413.

[49] G. Degrassi, P. Gambino, and G. F. Giudice, $B \rightarrow X_{s} \gamma$ in supersymmetry: Large contributions beyond the leading order, JHEP 12 (2000) 009, hep-ph/0009337.

[50] M. Carena, D. Garcia, U. Nierste, and C. E. M. Wagner, $b \rightarrow s \gamma$ and supersymmetry with large $\tan \beta$, Phys. Lett. B499 (2001) 141-146, hep-ph/0010003.

[51] C. Bobeth, M. Misiak, and J. Urban, Photonic penguins at two loops and $m_{t}$-dependence of $\operatorname{BR}\left(B \rightarrow X_{s} \ell^{+} \ell^{-}\right)$, Nucl. Phys. B574 (2000) 291-330, hep-ph/9910220.

[52] H. H. Asatryan, H. M. Asatrian, C. Greub, and M. Walker, Calculation of two loop virtual corrections to $b \rightarrow s \ell^{+} \ell^{-}$in the standard model, Phys. Rev. D65 (2002) 074004, hep-ph/0109140.

[53] H. H. Asatryan, H. M. Asatrian, C. Greub, and M. Walker, Complete gluon bremsstrahlung corrections to the process $b \rightarrow s \ell^{+} \ell^{-}$, Phys. Rev. D66 (2002) 034009, hep-ph/0204341.

[54] A. Ghinculov, T. Hurth, G. Isidori, and Y. P. Yao, The rare decay $B \rightarrow X_{s} \ell^{+} \ell^{-}$to $N N L L$ precision for arbitrary dilepton invariant mass, Nucl. Phys. B685 (2004) 351-392, hep-ph/0312128.

[55] P. Gambino, M. Gorbahn, and U. Haisch, Anomalous dimension matrix for radiative and rare semileptonic B decays up to three loops, Nucl. Phys. B673 (2003) 238-262, hep-ph/0306079.

[56] C. Bobeth, P. Gambino, M. Gorbahn, and U. Haisch, Complete NNLO QCD analysis of $\bar{B} \rightarrow X_{s} \ell^{+} \ell^{-}$and higher order electroweak effects, JHEP 04 (2004) 071, hep-ph/0312090.

[57] T. Huber, E. Lunghi, M. Misiak, and D. Wyler, Electromagnetic Logarithms in $B \rightarrow X_{s} \ell^{+} \ell^{-}$, Nucl. Phys. B740 (2006) 105-137, hep-ph/0512066.

[58] M. Beneke, T. Feldmann, and D. Seidel, Systematic approach to exclusive $B \rightarrow V \ell^{+} \ell^{-}, V \gamma$ decays, Nucl. Phys. B612 (2001) 25-58, hep-ph/0106067.

[59] M. Beneke, T. Feldmann, and D. Seidel, Exclusive radiative and electroweak $b \rightarrow d$ and $b \rightarrow s$ penguin decays at NLO, Eur. Phys. J. C41 (2005) 173-188, hep-ph/0412400.

[60] M. Misiak, The $b \rightarrow s e^{+} e^{-}$and $b \rightarrow s \gamma$ decays with next-to-leading logarithmic QCD corrections, Nucl. Phys. B393 (1993) 23-45.

[61] A. J. Buras and M. Münz, Effective Hamiltonian for $B \rightarrow X_{s} e^{+} e^{-}$beyond leading logarithms in the NDR and HV schemes, Phys. Rev. D52 (1995) 186-195, hep-ph/9501281.

[62] G. Hiller and F. Krüger, More model-independent analysis of $b \rightarrow s$ processes, Phys. Rev. D69 (2004) 074020, hep-ph/0310219.

[63] G. Buchalla, A. J. Buras, and M. E. Lautenbacher, Weak decays beyond leading logarithms, Rev. Mod. Phys. 68 (1996) 1125-1144, hep-ph/9512380. See also references therein. 
[64] C. Bobeth, A. J. Buras, and T. Ewerth, $\bar{B} \rightarrow X_{s} \ell^{+} \ell^{-}$in the MSSM at NNLO, Nucl. Phys. B713 (2005) 522-554, hep-ph/0409293.

[65] A. Ali, E. Lunghi, C. Greub, and G. Hiller, Improved model-independent analysis of semileptonic and radiative rare B decays, Phys. Rev. D66 (2002) 034002, hep-ph/0112300].

[66] G. Burdman, Short distance coefficients and the vanishing of the lepton asymmetry in $B \rightarrow V \ell^{+} \ell^{-}$, Phys. Rev. D57 (1998) 4254-4257, hep-ph/9710550].

[67] H. H. Asatrian, H. M. Asatrian, C. Greub, and M. Walker, Two-loop virtual corrections to $B \rightarrow X_{s} \ell^{+} \ell^{-}$in the Standard Model, Phys. Lett. B507 (2001) 162-172, hep-ph/0103087.

[68] H. M. Asatrian, K. Bieri, C. Greub, and A. Hovhannisyan, NNLL corrections to the angular distribution and to the forward-backward asymmetries in $B \rightarrow X_{s} \ell^{+} \ell^{-}$, Phys. Rev. D66 (2002) 094013, hep-ph/0209006.

[69] Belle Collaboration, M. Iwasaki et. al., Improved measurement of the electroweak penguin process $B \rightarrow X_{s} \ell^{+} \ell^{-}$, Phys. Rev. D72 (2005) 092005, hep-ex/0503044.

[70] BaBar Collaboration, B. Aubert et. al., Measurement of the $B \rightarrow X_{s} \ell^{+} \ell^{-}$branching fraction with a sum over exclusive modes, Phys. Rev. Lett. 93 (2004) 081802, hep-ex/0404006.

[71] A. Ishikawa et. al., Measurement of forward-backward asymmetry and Wilson coefficients in $B \rightarrow K^{*} \ell^{+} \ell^{-}$, Phys. Rev. Lett. 96 (2006) 251801, hep-ex/0603018.

[72] E. Lunghi, W. Porod, and O. Vives, Analysis of enhanced $\tan (\beta)$ corrections in MFV GUT scenarios, Phys. Rev. D74 (2006) 075003, hep-ph/0605177.

[73] A. J. Buras, A. Poschenrieder, M. Spranger, and A. Weiler, The impact of universal extra dimensions on $B \rightarrow X_{s} \gamma, B \rightarrow X_{s}$ gluon, $B \rightarrow X_{s} \mu^{+} \mu^{-}, K_{L} \rightarrow \pi^{0} e^{+} e^{-}$, and $\epsilon^{\prime} / \epsilon$, Nucl. Phys. B678 (2004) 455-490, hep-ph/0306158.

[74] K. Ikado, talk presented at FPCP 2006 (9-12 April 2006, Vancouver, Canada) http://fpcp2006.triumf.ca.

[75] Heavy Flavor Averaging Group: http://www.slac.stanford.edu/xorg/hfag.

[76] W.-M. Yao et. al., Review of Particle Physics, Journal of Physics G 33 (2006) 1+.

[77] S. Hashimoto, Recent results from lattice calculations, Int. J. Mod. Phys. A20 (2005) 5133-5144, hep-ph/0411126.

[78] K. Ikado et. al., Evidence of the purely leptonic decay $B^{-} \rightarrow \tau^{-} \bar{\nu}_{\tau}$, Phys. Rev. Lett. 97 (2006) 251802, hep-ex/0604018.

[79] BaBar Collaboration, B. Aubert et. al., A search for $B^{+} \rightarrow \tau^{+} \nu$ recoiling against $B^{-} \rightarrow D^{0} \ell^{-} \bar{\nu}_{\ell} X$, hep-ex/0608019.

[80] UTfit Collaboration, M. Bona et. al., The unitarity triangle fit in the standard model and hadronic parameters from lattice QCD: A reappraisal after the measurements of $\Delta m_{s}$ and $B R\left(B \rightarrow \tau \nu_{\tau}\right)$, JHEP 10 (2006) 081, hep-ph/0606167.

[81] W.-S. Hou, Enhanced charged Higgs boson effects in $B^{-} \rightarrow \tau \bar{\nu}, \mu \bar{\nu}$ and $b \rightarrow \tau \bar{\nu}+X$, Phys. Rev. D48 (1993) 2342-2344.

[82] A. G. Akeroyd and S. Recksiegel, The effect of $H^{ \pm}$on $B^{ \pm} \rightarrow \tau^{ \pm} \nu_{\tau}$ and $B^{ \pm} \rightarrow \mu^{ \pm} \nu_{\mu}, J$. Phys. G29 (2003) 2311-2317, hep-ph/0306037. 
[83] G. Isidori and P. Paradisi, Hints of large $\tan (\beta)$ in flavour physics, Phys. Lett. B639 (2006) 499-507, hep-ph/0605012.

[84] J. P. Miller, E. de Rafael, and B. L. Roberts, Muon $g-2$ : Review of Theory and Experiment, Rept. Prog. Phys. 70 (2007) 795, hep-ph/0703049].

[85] Muon $(g-2)$ Collaboration, G. W. Bennett et. al., Final report of the muon E821 anomalous magnetic moment measurement at BNL, Phys. Rev. D73 (2006) 072003, hep-ex/0602035.

[86] K. Hagiwara, A. D. Martin, D. Nomura, and T. Teubner, Improved predictions for $g-2$ of the muon and $\alpha_{\mathrm{QED}}\left(M_{Z}^{2}\right)$, hep-ph/0611102.

[87] M. Czakon, U. Haisch, and M. Misiak, Four-loop anomalous dimensions for radiative flavour- changing decays, JHEP 03 (2007) 008, [hep-ph/0612329]. See also references therein.

[88] M. Ciuchini, G. Degrassi, P. Gambino, and G. F. Giudice, Next-to-leading QCD corrections to $B \rightarrow X_{s} \gamma$ in Supersymmetry, Nucl. Phys. B534 (1998) 3-20, hep-ph/9806308.

[89] F. Borzumati, C. Greub, and Y. Yamada, Beyond leading-order corrections to $\bar{B} \rightarrow X_{s} \gamma$ at large $\tan \beta$ : The charged-Higgs contribution, Phys. Rev. D69 (2004) 055005, hep-ph/0311151.

[90] G. Degrassi, P. Gambino, and P. Slavich, QCD corrections to radiative B decays in the MSSM with minimal flavor violation, Phys. Lett. B635 (2006) 335-342, hep-ph/0601135.

[91] P. Gambino, U. Haisch, and M. Misiak, Determining the sign of the $b \rightarrow$ s $\gamma$ amplitude, Phys. Rev. Lett. 94 (2005) 061803, hep-ph/0410155.

[92] See the CERNlib website: http://cernlib.web.cern.ch/cernlib/.

[93] M. C. Gonzalez-Garcia and M. Maltoni, Phenomenology with Massive Neutrinos, 0704.1800 .

[94] T. Blažek, R. Dermíšek, and S. Raby, Predictions for Higgs and SUSY spectra from SO(10) Yukawa unification with $\mu>0$, Phys. Rev. Lett. 88 (2002) 111804, hep-ph/0107097.

[95] T. Blažek, R. Dermíšek, and S. Raby, Yukawa unification in SO(10), Phys. Rev. D65 (2002) 115004, hep-ph/0201081.

[96] D. Auto et. al., Yukawa coupling unification in supersymmetric models, JHEP 06 (2003) 023, hep-ph/0302155.

[97] J. A. Bagger, J. L. Feng, N. Polonsky, and R.-J. Zhang, Superheavy supersymmetry from scalar mass A-parameter fixed points, Phys. Lett. B473 (2000) 264-271, hep-ph/9911255.

[98] R. Dermíšek, S. Raby, L. Roszkowski, and R. Ruiz de Austri, Dark matter and $B_{s} \rightarrow \mu^{+} \mu^{-}$ with minimal SO(10) soft SUSY breaking, JHEP 04 (2003) 037, hep-ph/0304101.

[99] R. Dermíšek, S. Raby, L. Roszkowski, and R. Ruiz de Austri, Dark matter and $B_{s} \rightarrow \mu^{+} \mu^{-}$ with minimal SO(10) soft SUSY breaking II, JHEP 09 (2005) 029, hep-ph/0507233.

[100] See talk by A. Maciel at HEP 2007, Parallel Session "Flavour Physics and CP Violation", July 20, 2007.

[101] See talk by G. De Nardo at SUSY 2007, Parallel Session "Flavour Physics", July 27, 2007. 


\begin{tabular}{|l|l|l|l|l|}
\hline$m_{16}$ & 4000 & 6000 & 10000 & 4000 \\
$\mu$ & 378 & 953 & 1200 & -2106 \\
\hline \hline$M_{1 / 2}$ & 147.3 & 145.6 & 146.7 & 229.9 \\
$A_{0}$ & -7787.4 & -11924.0 & -20070.0 & -630.13 \\
$\tan \beta$ & 49.9 & 48.8 & 48.7 & 49.3 \\
$1 / \alpha_{G}$ & 24.7 & 24.7 & 24.7 & 24.6 \\
$M_{G} / 10^{16}$ & 2.45 & 3.11 & 4.8 & 4.96 \\
$\epsilon_{3} / \%$ & -3.81 & -4.12 & -5.37 & -5.6 \\
$\left(m_{H_{u}} / m_{16}\right)^{2}$ & 1.59 & 1.55 & 1.57 & 0.52 \\
$\left(m_{H_{d}} / m_{16}\right)^{2}$ & 1.86 & 1.79 & 1.8 & 1.0 \\
$M_{R_{1}} / 10^{10}$ & 1.059 & 1.05 & 1.072 & 0.8999 \\
$M_{R_{2}} / 10^{10}$ & -74.85 & -70.51 & -71.93 & -66.94 \\
$M_{R_{3}} / 10^{10}$ & 3244.0 & 3053.0 & 3069.0 & 2718.0 \\
$\lambda$ & 0.618 & 0.583 & 0.582 & 0.578 \\
$\epsilon$ & 0.0473 & 0.048 & 0.0477 & 0.048 \\
$\epsilon^{\prime}$ & -0.0034 & -0.00338 & -0.00342 & -0.00356 \\
$|\rho|$ & 0.0566 & 0.0584 & 0.0574 & 0.0567 \\
$|\sigma|$ & 1.02 & 1.03 & 1.03 & 0.987 \\
$|\tilde{\epsilon}|$ & 0.00964 & 0.00957 & 0.00967 & 0.00999 \\
$|\xi|$ & 0.134 & 0.134 & 0.134 & 0.14 \\
$\arg \rho$ & 3.93 & 3.92 & 3.94 & 3.94 \\
$\arg \sigma$ & 0.641 & 0.617 & 0.622 & 0.751 \\
$\arg \tilde{\epsilon}$ & 0.484 & 0.492 & 0.491 & 0.505 \\
$\arg \xi$ & 3.61 & 3.6 & 3.61 & 3.58 \\
\hline
\end{tabular}

Table 6: Input parameters for the fits presented in section 7.2 (cf. Table 1). Dimensionful quantities are given in units of $\mathrm{GeV}$. 


\begin{tabular}{|l|l|l|l|}
\hline Observable & Exp. value & Fit value & Pull $(\sigma)$ \\
\hline \hline$M_{W}$ & 80.403 & 80.4 & 0.1 \\
$M_{Z}$ & 91.1876 & 90.6 & 1.3 \\
$G_{\mathrm{F}} \times 10^{5}$ & 1.16637 & 1.16 & 0.7 \\
$1 / \alpha_{\mathrm{em}}$ & 137.036 & 136.4 & 0.9 \\
$\alpha_{s}\left(M_{Z}\right)$ & 0.1176 & 0.115 & 1.1 \\
$M_{t}$ & 170.9 & 171.4 & 0.2 \\
$m_{b}\left(m_{b}\right)$ & 4.2 & 4.31 & 1.5 \\
$m_{c}\left(m_{b}\right)$ & 1.25 & 1.15 & 1.2 \\
$m_{s}(2 \mathrm{GeV})$ & 0.095 & 0.107 & 0.5 \\
$m_{d}(2 \mathrm{GeV})$ & 0.005 & 0.00741 & 1.2 \\
$m_{u}(2 \mathrm{GeV})$ & 0.00225 & 0.00462 & $\mathbf{3 . 2}$ \\
$M_{\tau}$ & 1.777 & 1.77 & 0.4 \\
$M_{\mu}$ & 0.10566 & 0.106 & 0.1 \\
$M_{e}$ & 0.000511 & 0.000511 & 0.0 \\
$\left|V_{u s}\right|$ & 0.2258 & 0.225 & 0.6 \\
$\left|V_{u b}\right| \times 10^{3}$ & 4.1 & 3.26 & $\mathbf{2 . 1}$ \\
$\left|V_{c b}\right|$ & 0.0416 & 0.0417 & 0.1 \\
$\sin 2 \beta$ & 0.675 & 0.637 & 1.4 \\
$\Delta m_{31}^{2} \times 10^{21}$ & 2.6 & 2.6 & 0.0 \\
$\Delta m_{21}^{2} \times 10^{23}$ & 7.9 & 7.9 & 0.0 \\
$\sin 2 \theta_{12}$ & 0.852 & 0.85 & 0.1 \\
$\sin 2 \theta_{23}$ & 0.996 & 1.0 & 0.2 \\
$\epsilon_{K} \times 10^{3}$ & 2.229 & 2.32 & 0.4 \\
\hline $\mathrm{BR}\left(B \rightarrow X_{s} \gamma\right) \times 10^{4}$ & 3.55 & 0.885 & $\mathbf{5 . 0}$ \\
$\mathrm{BR}\left(B \rightarrow X_{s} \ell^{+} \ell^{-}\right) \times 10^{6}$ & 1.6 & 1.8 & 0.3 \\
$\Delta M_{s} / \Delta M_{d}$ & 35.05 & 29.8 & 1.4 \\
$\mathrm{BR}\left(B^{+} \rightarrow \tau^{+} \nu\right) \times 10^{4}$ & 1.31 & 0.336 & $\mathbf{2 . 0}$ \\
\hline & & total $\chi^{2}:$ & $\mathbf{5 8 . 3}$ \\
\hline
\end{tabular}

Table 7: Fit results for the case $m_{16}=4 \mathrm{TeV}, \mu=378 \mathrm{GeV}$. The pull for the $i^{\text {th }}$ observable represents the square root of the corresponding entry in the $\chi^{2}$ function (7.1). Corresponding predictions are reported in Table 11. Dimensionful quantities are given in units of GeV. 


\begin{tabular}{|c|c|c|c|}
\hline Observable & Exp. value & Fit value & $\overline{\text { Pull }(\sigma)}$ \\
\hline$M_{W}$ & 80.403 & 80.5 & 0.2 \\
\hline$M_{Z}$ & 91.1876 & 90.6 & 1.2 \\
\hline$G_{\mathrm{F}} \times 10^{5}$ & 1.16637 & 1.16 & 0.5 \\
\hline $1 / \alpha_{\mathrm{em}}$ & 137.036 & 136.5 & 0.8 \\
\hline$\alpha_{s}\left(M_{Z}\right)$ & 0.1176 & 0.116 & 0.5 \\
\hline$M_{t}$ & 170.9 & 169.8 & 0.6 \\
\hline$m_{b}\left(m_{b}\right)$ & 4.2 & 4.29 & 1.3 \\
\hline$m_{c}\left(m_{b}\right)$ & 1.25 & 1.14 & 1.2 \\
\hline$m_{s}(2 \mathrm{GeV})$ & 0.095 & 0.106 & 0.4 \\
\hline$m_{d}(2 \mathrm{GeV})$ & 0.005 & 0.00727 & 1.1 \\
\hline$m_{u}(2 \mathrm{GeV})$ & 0.00225 & 0.00465 & 3.2 \\
\hline$M_{\tau}$ & 1.777 & 1.77 & 0.3 \\
\hline$M_{\mu}$ & 0.10566 & 0.106 & 0.1 \\
\hline$M_{e}$ & 0.000511 & 0.000511 & 0.0 \\
\hline$\left|V_{u s}\right|$ & 0.2258 & 0.225 & 0.6 \\
\hline$\left|V_{u b}\right| \times 10^{3}$ & 4.1 & 3.26 & 2.1 \\
\hline$\left|V_{c b}\right|$ & 0.0416 & 0.0417 & 0.1 \\
\hline $\sin 2 \beta$ & 0.675 & 0.638 & 1.4 \\
\hline$\Delta m_{31}^{2} \times 10^{21}$ & 2.6 & 2.6 & 0.0 \\
\hline$\Delta m_{21}^{2} \times 10^{23}$ & 7.9 & 7.9 & 0.0 \\
\hline $\sin ^{2} 2 \theta_{12}$ & 0.852 & 0.852 & 0.0 \\
\hline $\sin ^{2} 2 \theta_{23}$ & 0.996 & 0.997 & 0.1 \\
\hline$\epsilon_{K} \times 10^{3}$ & 2.229 & 2.31 & 0.3 \\
\hline $\operatorname{BR}\left(B \rightarrow X_{s} \gamma\right) \times 10^{4}$ & 3.55 & 2.34 & 2.3 \\
\hline $\mathrm{BR}\left(B \rightarrow X_{s} \ell^{+} \ell^{-}\right) \times 10^{6}$ & 1.6 & 1.62 & 0.0 \\
\hline$\Delta M_{s} / \Delta M_{d}$ & 35.05 & 30.0 & 1.4 \\
\hline $\mathrm{BR}\left(B^{+} \rightarrow \tau^{+} \nu\right) \times 10^{4}$ & 1.31 & 0.398 & 1.9 \\
\hline \multicolumn{4}{|c|}{ total $\chi^{2}$ : } \\
\hline
\end{tabular}

Table 8: Fit results for the case $m_{16}=6 \mathrm{TeV}, \mu=953 \mathrm{GeV}$. Corresponding predictions are reported in Table 11. Dimensionful quantities are given in units of $\mathrm{GeV}$. 


\begin{tabular}{|c|c|c|c|}
\hline Observable & Exp. value & Fit value & $\overline{\text { Pull }(\sigma)}$ \\
\hline$M_{W}$ & 80.403 & 80.6 & 0.5 \\
\hline$M_{Z}$ & 91.1876 & 90.7 & 1.1 \\
\hline$G_{\mathrm{F}} \times 10^{5}$ & 1.16637 & 1.16 & 0.3 \\
\hline $1 / \alpha_{\mathrm{em}}$ & 137.036 & 136.8 & 0.4 \\
\hline$\alpha_{s}\left(M_{Z}\right)$ & 0.1176 & 0.117 & 0.2 \\
\hline$M_{t}$ & 170.9 & 170.6 & 0.2 \\
\hline$m_{b}\left(m_{b}\right)$ & 4.2 & 4.22 & 0.3 \\
\hline$m_{c}\left(m_{b}\right)$ & 1.25 & 1.14 & 1.2 \\
\hline$m_{s}(2 \mathrm{GeV})$ & 0.095 & 0.107 & 0.5 \\
\hline$m_{d}(2 \mathrm{GeV})$ & 0.005 & 0.00741 & 1.2 \\
\hline$m_{u}(2 \mathrm{GeV})$ & 0.00225 & 0.00461 & 3.1 \\
\hline$M_{\tau}$ & 1.777 & 1.78 & 0.1 \\
\hline$M_{\mu}$ & 0.10566 & 0.106 & 0.1 \\
\hline$M_{e}$ & 0.000511 & 0.000511 & 0.0 \\
\hline$\left|V_{u s}\right|$ & 0.2258 & 0.225 & 0.6 \\
\hline$\left|V_{u b}\right| \times 10^{3}$ & 4.1 & 3.26 & 2.1 \\
\hline$\left|V_{c b}\right|$ & 0.0416 & 0.0416 & 0.1 \\
\hline $\sin 2 \beta$ & 0.675 & 0.639 & 1.4 \\
\hline$\Delta m_{31}^{2} \times 10^{21}$ & 2.6 & 2.6 & 0.0 \\
\hline$\Delta m_{21}^{2} \times 10^{23}$ & 7.9 & 7.9 & 0.0 \\
\hline $\sin ^{2} 2 \theta_{12}$ & 0.852 & 0.852 & 0.0 \\
\hline $\sin ^{2} 2 \theta_{23}$ & 0.996 & 1.0 & 0.2 \\
\hline$\epsilon_{K} \times 10^{3}$ & 2.229 & 2.33 & 0.4 \\
\hline $\operatorname{BR}\left(B \rightarrow X_{s} \gamma\right) \times 10^{4}$ & 3.55 & 2.86 & 1.3 \\
\hline $\mathrm{BR}\left(B \rightarrow X_{s} \ell^{+} \ell^{-}\right) \times 10^{6}$ & 1.6 & 1.62 & 0.0 \\
\hline$\Delta M_{s} / \Delta M_{d}$ & 35.05 & 31.1 & 1.1 \\
\hline $\mathrm{BR}\left(B^{+} \rightarrow \tau^{+} \nu\right) \times 10^{4}$ & 1.31 & 0.517 & 1.7 \\
\hline \multicolumn{4}{|c|}{ total $\chi^{2}:$} \\
\hline
\end{tabular}

Table 9: Fit results for the case $m_{16}=10 \mathrm{TeV}, \mu=1200 \mathrm{GeV}$. Corresponding predictions are reported in Table 11. Dimensionful quantities are given in units of $\mathrm{GeV}$. 


\begin{tabular}{|l|l|l|l|}
\hline Observable & Exp. value & Fit value & Pull $(\sigma)$ \\
\hline \hline$M_{W}$ & 80.403 & 80.7 & 0.7 \\
$M_{Z}$ & 91.1876 & 90.7 & 1.1 \\
$G_{\mathrm{F}} \times 10^{5}$ & 1.16637 & 1.17 & 0.2 \\
$1 / \alpha_{\mathrm{em}}$ & 137.036 & 136.9 & 0.3 \\
$\alpha_{s}\left(M_{Z}\right)$ & 0.1176 & 0.118 & 0.0 \\
$M_{t}$ & 170.9 & 170.5 & 0.2 \\
$m_{b}\left(m_{b}\right)$ & 4.2 & 4.19 & 0.1 \\
$m_{c}\left(m_{b}\right)$ & 1.25 & 1.14 & 1.2 \\
$m_{s}(2 \mathrm{GeV})$ & 0.095 & 0.0999 & 0.2 \\
$m_{d}(2 \mathrm{GeV})$ & 0.005 & 0.00716 & 1.1 \\
$m_{u}(2 \mathrm{GeV})$ & 0.00225 & 0.00446 & $\mathbf{3 . 0}$ \\
$M_{\tau}$ & 1.777 & 1.78 & 0.1 \\
$M_{\mu}$ & 0.10566 & 0.106 & 0.2 \\
$M_{e}$ & 0.000511 & 0.000511 & 0.1 \\
$\left|V_{u s}\right|$ & 0.2258 & 0.224 & 1.2 \\
$\left|V_{u b}\right| \times 10^{3}$ & 4.1 & 3.26 & $\mathbf{2 . 1}$ \\
$\left|V_{c b}\right|$ & 0.0416 & 0.0416 & 0.0 \\
$\sin 2 \beta$ & 0.675 & 0.64 & 1.3 \\
$\Delta m_{31}^{2} \times 10^{21}$ & 2.6 & 2.6 & 0.0 \\
$\Delta m_{21}^{2} \times 10^{23}$ & 7.9 & 7.9 & 0.0 \\
$\sin 2 \theta_{12}$ & 0.852 & 0.851 & 0.0 \\
$\sin 2 \theta_{23}$ & 0.996 & 0.996 & 0.0 \\
$\epsilon_{K} \times 10^{3}$ & 2.229 & 2.35 & 0.5 \\
\hline $\mathrm{BR}\left(B \rightarrow X_{s} \gamma\right) \times 10^{4}$ & 3.55 & 3.34 & 0.4 \\
$\mathrm{BR}\left(B \rightarrow X_{s} \ell^{+} \ell^{-}\right) \times 10^{6}$ & 1.6 & 1.63 & 0.0 \\
$\Delta M_{s} / \Delta M_{d}$ & 35.05 & 31.4 & 1.0 \\
$\mathrm{BR}\left(B^{+} \rightarrow \tau^{+} \nu\right) \times 10^{4}$ & 1.31 & 0.59 & 1.5 \\
\hline & & total $\chi^{2}:$ & $\mathbf{2 4 . 5}$ \\
\hline & & & \\
\hline
\end{tabular}

Table 10: Fit results for the case $m_{16}=4 \mathrm{TeV}, \mu=-2106 \mathrm{GeV}$. Corresponding predictions are reported in Table 11. Dimensionful quantities are given in units of $\mathrm{GeV}$. 


\begin{tabular}{|l|l|l|l|l|}
\hline$m_{16}$ & 4000 & 6000 & 10000 & 4000 \\
$\mu$ & 378 & 953 & 1200 & -2106 \\
\hline \hline $\mathrm{BR}\left(B_{s} \rightarrow \mu^{+} \mu^{-}\right) \times 10^{8}$ & 8.6 & 7.7 & 2.1 & 0.33 \\
$\hat{s}_{0}$ & 0.022 & 0.13 & 0.14 & 0.16 \\
$\mathrm{BR}(\mu \rightarrow e \gamma) \times 10^{13}$ & 0.36 & 0.021 & 0.0026 & 0.011 \\
$\delta a_{\mu}^{\text {SUSY }} \times 10^{10}$ & +5.8 & +1.6 & +0.52 & -2.9 \\
\hline$M_{h_{0}}$ & 126 & 129 & 129 & 119 \\
$M_{A}$ & 507 & 559 & 842 & 1800 \\
$m_{\tilde{t}_{1}}$ & 640 & 1172 & 1903 & 2627 \\
$m_{\tilde{b}_{1}}$ & 895 & 1475 & 2366 & 2488 \\
$m_{\tilde{\tau}_{1}}$ & 1510 & 2419 & 3933 & 2931 \\
$m_{\tilde{\chi}_{1}^{0}}$ & 60 & 60 & 60 & 94 \\
$m_{\tilde{\chi}_{1}^{+}}$ & 115 & 119 & 120 & 189 \\
$m_{\tilde{g}}$ & 462 & 478 & 506 & 703 \\
\hline \multicolumn{4}{|l}{}
\end{tabular}

Table 11: Predictions for the scenarios presented in section 7.2. Masses are given in units of GeV. $\hat{s}_{0}$ is the zero position of the forward-backward asymmetry in $B \rightarrow X_{s} \ell^{+} \ell^{-}$(cf. Section 5.4). The quantity $\delta a_{\mu}^{\text {SUSY }}$ is the SUSY contribution to $a_{\mu} \equiv(g-2)_{\mu} / 2$, which is currently measured to be $+27.6 \times 10^{-10}$ larger than the SM prediction 85, 86, with an uncertainty of about $8 \times 10^{-10}$. 